\title{
Complex Dynamics Induced by Asymmetry in Coupled Laser Systems
}

\author{
A. Bountis, Y. Kominis, J.Shena, V. Kovanis
}

Coupled semiconductor lasers are systems possessing complex dynamics, which makes them interesting for many applications in photonics. In this paper, we first review our results on the existence and stability of asymmetric phase-locked states of a single dimer consisting of two coupled semiconductor lasers. We show that stable phase-locked states of arbitrary asymmetry exist, whose field amplitude ratio and phase difference can be dynamically controlled by appropriate electronic current injection. Moreover, we obtain stable limit cycles with asymmetric characteristics, emerging through Hopf bifurcations from these phase-locked states. Also, we emphasize the importance of exceptional points, and we show that asymmetry enables their existence in extended regions of parameter space. The dynamics of asymmetric dimers under small signal modulation of the pumping current is also investigated and the occurrence of antiresonances and sharp resonances with very high frequencies is demonstrated. Finally, we describe

Received September 22, 2019

Accepted November 18, 2019

This research is partly supported by the ORAU grant entitled "Taming Chimeras to Achieve the Superradiant Emitter", funded by Nazarbayev University, Republic of Kazakhstan. This work was partially also supported by the Ministry of Education and Science of the Republic of Kazakhstan via Contract No. 339/76-2015.

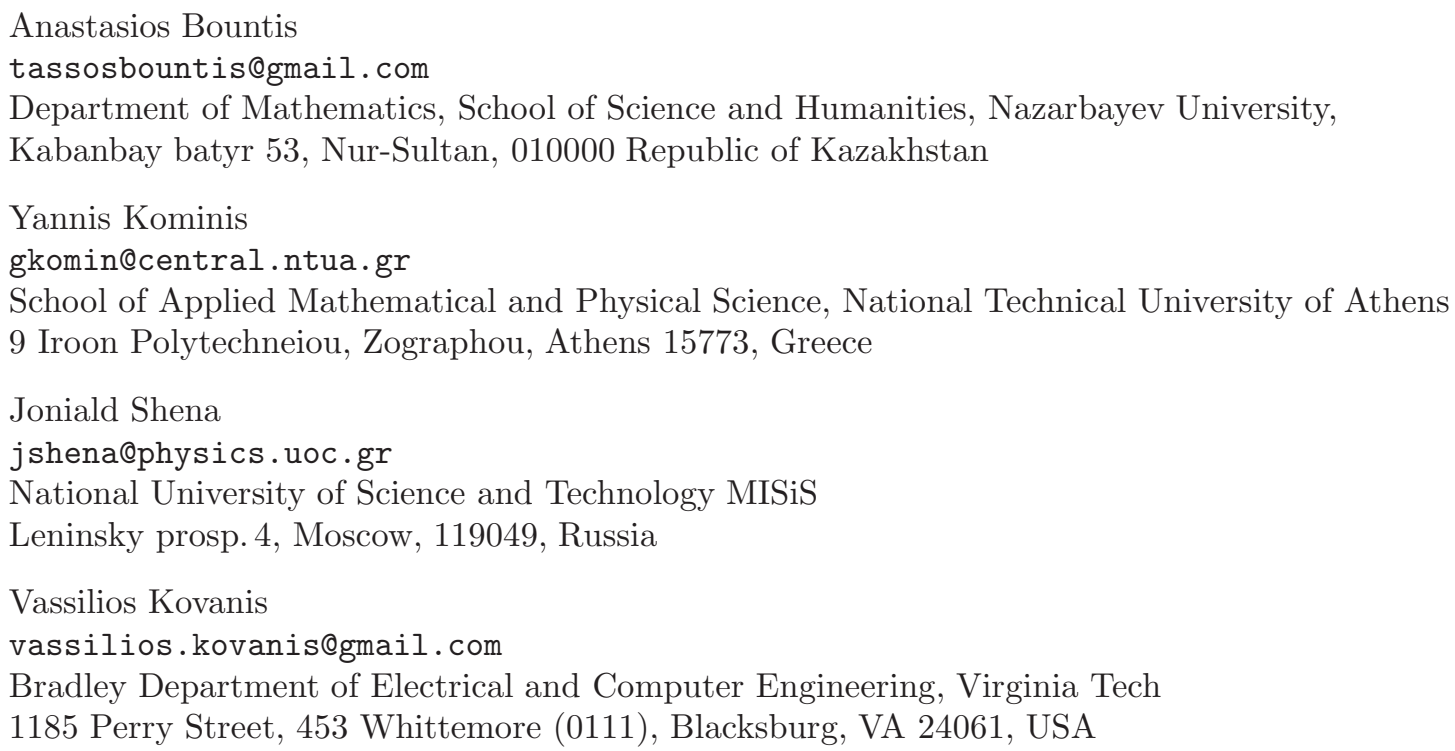

RUSSIAN JOURNAL OF NONLINEAR DYNAMICS, 2019, 15(4), 429-455 
our recent findings on optically coupled arrays of coupled dimers and explore their fascinating nonlinear dynamics. In particular, we couple in an appropriate way a large number of dimers and show that, depending on their degree of asymmetry, they exhibit organized high amplitude oscillations, or oscillate very close to phase-locked states, suggesting that such photonic networks may prove useful in a variety of beam forming and beam shaping applications.

Keywords: coupled mode equations, semiconductor laser arrays, Hopf bifurcations, limit cycle oscillations, coherence in photonic arrays

\section{Introduction}

Semiconductor laser arrays are photonic structures with great potential for applications in optical communications, sensing and imaging. One of their most attractive features is their electronically controlled operation and tunability, which enables them to transform current distributions into desirable field patterns. In that sense, a pair of coupled semiconductor lasers, herein called a dimer, constitutes a fundamental photonic oscillator, from which larger and more complicated structures can be built. The properties of such dimers are determined mostly by their stationary states, stability and bifurcations that can be controlled by current injection in the two lasers. The existence of stable asymmetric phase-locked states with unequal field amplitudes and phase differences crucially determines the dimer's far field patterns $[1,2]$ and its capability to act as a unit for synthesizing larger controllable active structures characterized by complex dynamics [3-10]. In addition to beam shaping applications [11], a pair of coupled lasers can also be considered as part of a "photonic processor" $[12,13]$.

A pair of coupled lasers, therefore, constitutes an excellent model for non-Hermitian optics that is of much current research interest. Its dynamics is accurately described by coupled mode equations for the complex field amplitudes [14-18], and has been extensively studied in (paritytime) $P T$-symmetric configurations [19-24]. The essential requirement for $P T$-symmetry is that there be no detuning between the two lasers, while in most cases gain and loss are exactly balanced. However, these conditions can be relaxed to allow for nonzero detuning and arbitrary gain or loss $[14,17]$. There are cases, however, where deviations from exact $P T$-symmetry are necessitated by practical reasons [17] or intentionally designed to obtain highly desirable stable nonlinear supermodes $[27,28]$.

When writing down coupled mode equations to study the dynamics of $P T$-symmetric lasers, one commonly neglects the nonlinearity of the system due to the coupling between field amplitudes and carrier densities. This approximation, however, excludes some important phenomena that are of great interest to photonics applications, such as the existence of symmetric and asymmetric phase-locked states, limit cycles [29,30] and localized synchronization effects [31, 32] that arise only when carrier density dynamics is taken into account $[14,29]$. Indeed, the latter introduces current injection as a control mechanism that can seriously affect the dynamics of the system.

In our earlier work, we began by studying the dynamics of a single laser dimer. We investigated in detail the existence and stability of its asymmetric phase-locked modes, as well as the role of detuning and inhomogeneous pumping between the lasers [37]. Remarkably, for zero detuning, we discovered stable asymmetric modes even when the two lasers are homogeneously pumped, i.e., the lasers are absolutely symmetric! These modes bifurcate to stable limit cycles through Hopf bifurcations, while for nonzero detuning we obtain phase-locked modes with arbitrary power, amplitude ratio and phase difference for appropriate pumping and detuning values. 
These asymmetric states are stable for large regions of parameters, in contrast to common coupled oscillators (whose asymmetric states are usually unstable [33]) and are due to the inclusion of carrier density dynamics in the model.

In all cases, asymmetric states have carrier densities with gain coefficients of opposite sign in each laser, so that the respective electric fields experience gain and loss, as in the case of PT-symmetric configurations. However, we discovered that deviations from $P T$-symmetry, expressed by nonzero detuning, can lead to asymmetric phase-locked states of arbitrary field amplitude ratio and phase difference that are most promising for applications.

Exceptional (fixed) points (EP) are very special, since the vanishing of imaginary parts in their eigenvalue spectrum leads to the collapse of eigenstates that has important consequences for the model's physical behavior. For a long time the existence of EP had been attributed to PTsymmetry, in simplified coupled mode equation models free from carrier-induced nonlinearities and with zero frequency detuning $[17,22,24]$. In a recent paper, however, it was shown that an "effective" form of PT-symmetry can be achieved even for nonzero frequency detuning, by judiciously selecting unequal pumping rates in the coupled rate equations [35].

We have shown that EP also exist under asymmetric configurations, with substantial differential pumping and nonzero optical frequency detuning [36, 39]. This results in phase-locked states that are neither PT-symmetric nor "effectively" PT-symmetric in the above sense and thus significantly extends the range of options, when operating the system at an EP. We have thus been able to locate new EP for a wide range of coupling, frequency detuning and pumping rates, thus facilitating the selection of appropriate parameters, according to specific experimental constraints.

We have also explored the response induced by small signal modulation in a laser dimer, when stable phase-locked states of varying asymmetry are considered [40]. We have found that the extended stability regions of these asymmetric states can lead to frequency response with a rich set of qualitatively new features: An asymmetric amplitude response of the coupled laser system, sharp resonances and antiresonances, and the achievement of efficient stable modulation of the system at frequencies that are orders of magnitude higher than its free running relaxation frequency.

The design of photonically integrated arrays of laser dimers, consisting of large numbers of properly coupled photonic emitters, is very important for many applications. It is well known that operating such an array in a phase-locked state offers a highly effective method for beam shaping because it increases the output power and reduces the required lasing threshold. Recently, active microring resonator arrays have been theoretically and experimentally investigated. For example, as pointed out in [46], this richly nonlinear, non-Hermitian system displays a broad range of behaviors, from single edge-mode lasing to multimode emission within the bulk of the array.

We have thus begun, more recently, to study the collective behavior of a large array of semiconductor lasers with nearest-neighbor interactions and fixed boundary conditions at both ends. The crucial parameters for the observed dynamics are the pumping rates, the interlaser coupling and the optical frequency detuning between the lasers, all of which introduce realistic inhomogeneities and dispersion effects within each dimer. We first discovered that, for symmetric arrays where all dimers have equal pumping rates and internal coupling $\eta_{i n}$ just above Hopf bifurcation points, nearly equal amplitude oscillations (NEAO) of the electric fields occur, which persist even when the external coupling between nearest neighbors $\eta_{e x}$ is nearly equal to $\eta_{i n}$. We thus found interdimer coupling regimes with NEAO even in arrays of $M=30$ dimers and beyond, for zero detuning and in the absence of noise.

RUSSIAN JOURNAL OF NONLINEAR DYNAMICS, 2019, 15(4), 429-455 
We also studied various asymmetric settings where the choices of pumping rates differ from the symmetric case. Here, the important result is that many dimers still execute stable NEAO for similar ranges of internal and external coupling parameters as in the case of symmetry. What is particularly interesting, however, is that new oscillatory phenomena are also found, termed SOPLS, where some of the lasers perform small oscillations about the (unstable) phase-locked state. Finally, varying slightly the detuning and adding weak noise to each dimer, we find wide parameter regimes where all these oscillatory phenomena can be robustly observed, not only for fixed but also for periodic boundary conditions.

This paper is organized as follows: After introducing the basic modal equations and discussing the phase-locked states and limit cycle solutions for the system of two coupled semiconductor lasers in Section 2, we focus on the eigenvalue spectra of steady states and identify their exceptional points and Hopf bifurcations in Section 3. Next, in Section 4, we study the signatures of the spectral line shape and the modulation response of a two-laser system (dimer) in the presence of noise. In Section 5, we extend our results to the case of arrays of many laser dimers and discover remarkably coherent oscillatory phenomena, where some lasers execute large-amplitude oscillations, while others remain very close to the unstable phase-locked state. Finally, we close in Section 6 with our conclusions and preliminary results of ongoing investigations.

\section{Rate equations model for two coupled lasers: phase-locked states and limit cycles}

The dynamics of an array of $M$ evanescently coupled semiconductor lasers is governed by the following equations describing the complex amplitude of the normalized electric field $\mathcal{E}_{i}$ and the normalized excess carrier density $N_{i}$ of each laser:

$$
\begin{aligned}
\frac{d \mathcal{E}_{i}}{d t} & =(1-i \alpha) \mathcal{E}_{i} N_{i}+i \eta\left(\mathcal{E}_{i+1}+\mathcal{E}_{i-1}\right)+i \omega_{i} \mathcal{E}_{i} & \\
T \frac{d N_{i}}{d t} & =P_{i}-N_{i}-\left(1+2 N_{i}\right)\left|\mathcal{E}_{i}\right|^{2}, & i=1, \ldots, M,
\end{aligned}
$$

$\alpha$ being the linewidth enhancement factor, $\eta$ a normalized coupling constant, $P_{i}$ the normalized excess electrical pumping rate, $\omega_{i}$ the normalized optical frequency detuning from a common reference, $T$ the ratio of carrier to photon lifetimes, and $t$ the normalized time [29]. When they are uncoupled $(\eta=0)$, the lasers exhibit free running relaxation with frequencies

$$
\Omega_{i}=\sqrt{\frac{2 P_{i}}{T}}
$$

Since we generally study lasers that are inhomogeneously pumped (i.e., $P_{i} \neq P_{j}$ ), we shall define a reference value for $P$ and a corresponding frequency

$$
\Omega=\sqrt{\frac{2 P}{T}}
$$

and rescale Eqs. (2.1) as follows:

$$
\begin{array}{rlr}
\frac{d \mathcal{E}_{i}}{d \tau} & =(1-i \alpha) \mathcal{E}_{i} Z_{i}+i \Lambda\left(\mathcal{E}_{i+1}+\mathcal{E}_{i-1}\right)+i \Omega_{i} \mathcal{E}_{i} & \\
2 P \frac{d Z_{i}}{d \tau} & =P_{i}-\Omega Z_{i}-\left(1+2 \Omega Z_{i}\right)\left|\mathcal{E}_{i}\right|^{2}, & i=1, \ldots, M,
\end{array}
$$

with

$$
\tau \equiv \Omega t, \quad Z_{i} \equiv N_{i} / \Omega, \quad \Lambda \equiv \eta / \Omega, \quad \Omega_{i} \equiv \omega_{i} / \Omega
$$


In [37] we studied the existence and stability of asymmetric phase-locked states for a single dimer $(M=2)$ under symmetric and asymmetric electrical pumping. Introducing now the amplitude and phase of the complex electric field in each laser as $\mathcal{E}_{i}=X_{i} e^{i \theta_{i}}$, Eqs. (2.4) for $M=2$ become

$$
\begin{aligned}
\frac{d X_{1}}{d \tau} & =X_{1} Z_{1}-\Lambda X_{2} \sin \theta \\
\frac{d X_{2}}{d \tau} & =X_{2} Z_{2}+\Lambda X_{1} \sin \theta \\
\frac{d \theta}{d \tau} & =\Delta-\alpha\left(Z_{2}-Z_{1}\right)+\Lambda\left(\frac{X_{1}}{X_{2}}-\frac{X_{2}}{X_{1}}\right) \cos \theta, \\
2 P \frac{d Z_{1}}{d \tau} & =P_{1}-\Omega Z_{1}-\left(1+2 \Omega Z_{1}\right) X_{1}^{2}, \\
2 P \frac{d Z_{2}}{d \tau} & =P_{2}-\Omega Z_{2}-\left(1+2 \Omega Z_{2}\right) X_{2}^{2},
\end{aligned}
$$

where we have defined $\Delta=\Omega_{2}-\Omega_{1}$ as the detuning, $\theta=\theta_{2}-\theta_{1}$ as the phase difference of the electric fields and use $P=\left(P_{1}+P_{2}\right) / 2$ to define $\Omega$ as in Eq. (2.3). As a reference case, we consider a dimer with $\alpha=5, T=400$ typically used in experiments with $P=0.5$. We thus have $\Omega=5 \times 10^{-2}$ and a coupling $\eta$ in the range of $\left[10^{-5}, 1\right]$, which corresponds to a $\Lambda$ in the range $\left[0.5 \times 10^{-3}, 0.5 \times 10^{2}\right]$.

The equilibria of (2.6) are phase-locked states given by the solutions of the algebraic system obtained by setting the time derivatives in (2.6) equal to zero, whose (linear) stability is determined by the eigenvalues of the Jacobian of the system. For the case of zero detuning $(\Delta=0)$ and symmetric electrical pumping $\left(P_{1}=P_{2}=P_{0}\right)$, two phase-locked states are known analytically: $X_{1}=X_{2}=\sqrt{P_{0}}, Z_{1}=Z_{2}=0$ and $\theta=0, \pi$. The in-phase state $(\theta=0)$ is stable for $\eta>\alpha P_{0} /\left(1+2 P_{0}\right)$, and the out-of-phase state $(\theta=\pi)$ for $\eta<\left(1+2 P_{0}\right) / 2 \alpha T$ [29].

The phase difference $\theta$ and the electric field ratio $\rho \equiv X_{2} / X_{1}$ of a phase-locked state determine the intensity response of the system. The incoherent intensity is defined as the sum $S \equiv\left|\mathcal{E}_{1}\right|^{2}+\left|\mathcal{E}_{2}\right|^{2}=\left(1+\rho^{2}\right) X_{1}^{2}$, while the coherent intensity corresponds to a superposition of the electric fields $I \equiv\left|\mathcal{E}_{1}+\mathcal{E}_{2}\right|^{2}=\left(1+\rho^{2}+2 \rho \cos \theta\right) X_{1}^{2}$. The coherent intensity depends on the phase difference $\theta$, but does not take into account the spatial distance between the lasers. Considering for simplicity the lasers as two point sources at a distance $d$, the coherent intensity is given by $I_{\phi}=\left|\mathcal{E}_{1}+e^{i 2 \pi(d / \lambda) \sin \phi} \mathcal{E}_{2}\right|^{2}=\mathcal{E}_{2} \mid=\left[1+\rho^{2}+2 \rho \cos (\theta+2 \pi(d / \lambda) \sin \phi)\right] X_{1}^{2}$, where $\phi$ is the azimuthal angle measured from the direction normal to the distance between the lasers, while $\lambda$ is the wavelength [34]. It is clear that the asymmetry of the phase-locked state measured by $\rho, \theta$ and the geometric parameter $d / \lambda$ define a specific far-field pattern $I_{\phi}$ with desirable characteristics.

Since we cannot solve the above system analytically for the desired unknowns, we will analyze the converse problem: Given a phase-locked state with field amplitude ratio $\rho \equiv X_{2} / X_{1}$ and phase difference $(\theta)$, we solve the algebraic system obtained by setting the rhs of Eqs. (2.6) equal to zero and determine the steady-state carrier densities $\left(Z_{1,2}\right)$, the detuning $(\Delta)$ and pumping rates $\left(P_{1,2}\right)$ in terms of $\rho$ and $\theta$. Let us demonstrate our approach in the case of $\Delta=0$. It is easy to verify that for every $\rho$ there exists an equilibrium of the dynamical system $(2.6)$ related to a fixed phase difference $\theta$ by the expression

$$
\tan \theta=\frac{1}{\alpha} \frac{\rho^{2}-1}{\rho^{2}+1}
$$


while the full set of equations yields:

$$
\begin{aligned}
& Z_{1}=\Lambda \rho \sin \theta \\
& Z_{2}=-\frac{\Lambda}{\rho} \sin \theta \\
& P_{1}=X_{0}^{2}+\left(1+2 X_{0}^{2}\right) \Omega \Lambda \rho \sin \theta \\
& P_{2}=\rho^{2} X_{0}^{2}-\left(1+2 \rho^{2} X_{0}^{2}\right) \frac{\Omega \Lambda}{\rho} \sin \theta
\end{aligned}
$$

where we have defined $X_{0} \equiv X_{1}$.

\subsection{Symmetric dimers}

For the case of symmetric pumping $\left(P_{1}=P_{2}=P_{0}\right)$ and zero detuning $(\Delta=0)$, we find phase-locked states with field amplitude $X_{0}$ given by

$$
X_{0}^{2}=\frac{\Omega \Lambda \sin \theta\left(\rho^{2}+1\right)}{\rho\left[\left(\rho^{2}-1\right)-4 \Omega \Lambda \rho \sin \theta\right]},
$$

while the common pumping rate is expressed by

$$
P_{0}=X_{0}^{2}+\left(1+2 X_{0}^{2}\right) \Omega \Lambda \rho \sin \theta .
$$

Remarkably, asymmetric phase-locked states exist with arbitrary amplitude ratio $(\rho)$, even for the case of identical lasers! More interestingly, this state is stable over a large region of parameter space as shown in Fig. 1. Moreover, the asymmetric phase-locked states undergo a Hopf bifurcation that gives rise to stable limit cycles similar to those we encounter in the symmetric case [29], but with generally different oscillation amplitudes [32].

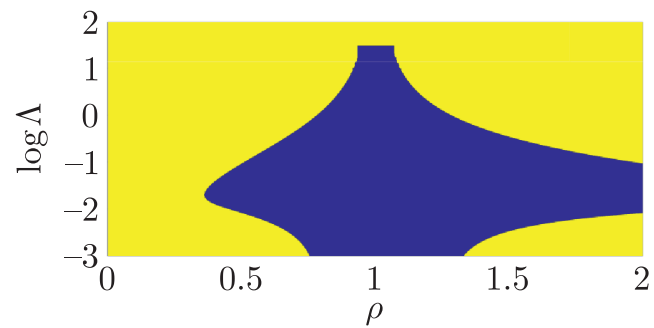

(a)

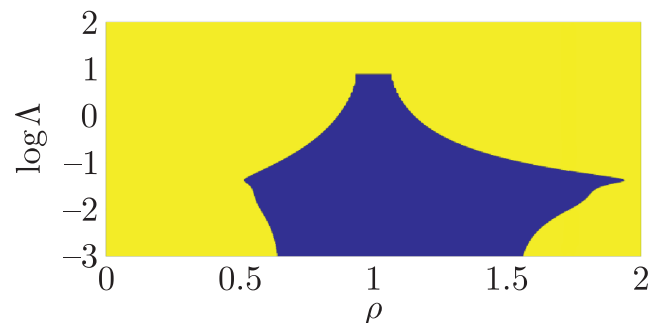

(b)

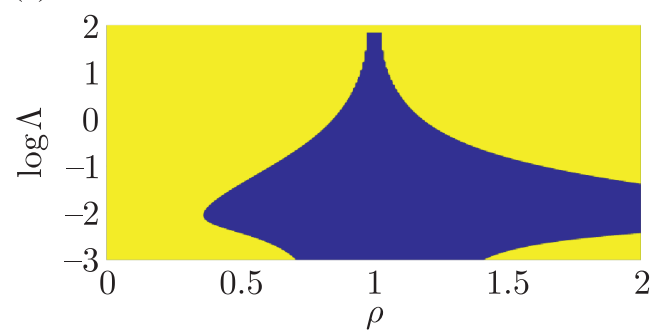

(c)

Fig. 1. Stability regions of asymmetric phase-locked states in a symmetric configuration with $\Delta=0$ and $P_{1}=P_{2}=P_{0}$ in the $(\Lambda, \rho)$ parameter space. Dark blue and light yellow areas correspond to stability and instability, respectively. (a) $\alpha=5$ and $T=400$, (b) $\alpha=1.5$ and $T=400$, (c) $\alpha=5$ and $T=2000$ (case of $[29]$ ). 


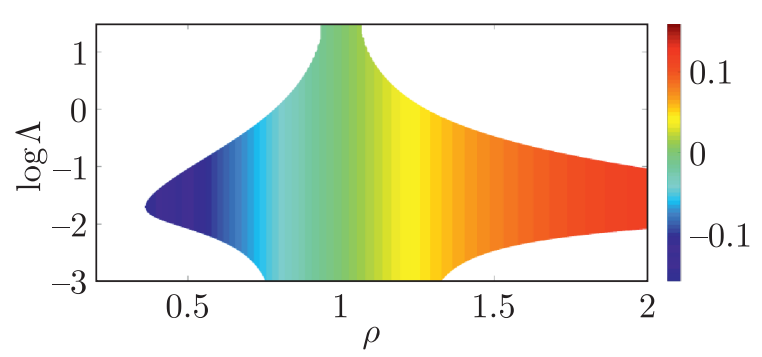

(a)

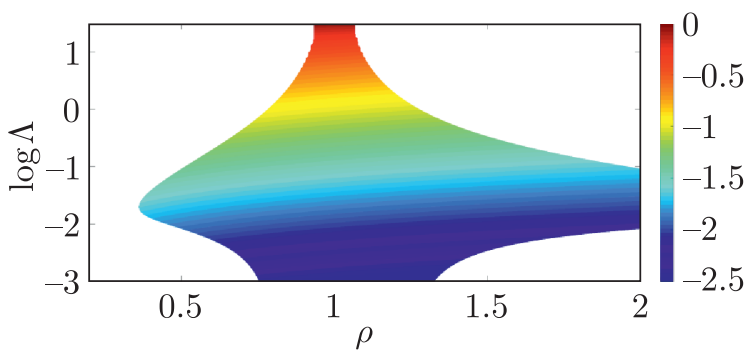

(b)

Fig. 2. Steady-state phase difference $\theta$ (a) and field amplitude $X_{0}$ (in logarithmic scale) (b) of the stable asymmetric phase-locked state in the $(\Delta, \rho)$ parameter space. Parameter values correspond to Fig. 1a.

Note how the amplitude ratio $\rho$ depends on the phase difference $\theta$, see Eq. (2.7) and Fig. 2a. Moreover, the field amplitude depends strongly on the ratio $\rho$ and the normalized coupling constant $\Lambda$ as shown in Eq. (2.10) and Fig. 2b. The pumping $P_{0}$ needed to have an asymmetric phase-locked state is given by Eq. (2.11), while the well-known symmetric states are obtained for $\rho=1[29]$.

We emphasize that phase-locked states with fixed phase difference $\theta$ but arbitrary amplitude $X_{0}$ also exist for different pumpings between the two lasers, given by Eq. (2.9). Their stability depends crucially on the electric field amplitude $X_{0}$. However, in comparison with Fig. 1a, for the same parameter values but with $P_{1}=P_{2}=P_{0}$, the extent of the stability regions is significantly reduced.

\subsection{Asymmetric dimers}

By analogy with the case of zero detuning we now exploit the analytical relations we have found connecting the steady-state carrier densities $\left(Z_{1,2}\right)$ with the appropriate detuning $(\Delta)$ and pumping rates $\left(P_{1,2}\right)$ parameters for arbitrary amplitude ratio $(\rho)$ and phase difference $(\theta)$, written in the form

$$
\begin{gathered}
Z_{1}=\Lambda \rho \sin \theta \\
Z_{2}=-\frac{\Lambda}{\rho} \sin \theta \\
\Delta=-\alpha \Lambda \sin \theta\left(\frac{1}{\rho}+\rho\right)-\Lambda \cos \theta\left(\frac{1}{\rho}-\rho\right), \\
P_{1}=X_{0}^{2}+\left(1+2 X_{0}^{2}\right) \Omega \Lambda \rho \sin \theta \\
P_{2}=\rho^{2} X_{0}^{2}-\left(1+2 \rho^{2} X_{0}^{2}\right) \frac{\Omega \Lambda}{\rho} \sin \theta .
\end{gathered}
$$

These relations imply that there always exists a phase-locked state with arbitrary asymmetry ratio $\rho$ and phase difference $\theta$, as long as the detuning $\Delta$ and the pumping rates $P_{1,2}$ obey Eqs. (2.13) and (2.14), respectively, with the steady-state carrier densities $\left(Z_{1,2}\right)$ given by Eqs. (2.12). These states exist over the whole parameter space and arbitrary amplitude $X_{0}$. The stability of these states depends strongly on the coupling $(\Lambda)$, the value of $\left(X_{0}\right)$ and the degree of asymmetry, characterized by $\rho$ and $\theta$, as shown in Figs. 3a-3d.

There is a variety of stable and unstable asymmetric phase-locked states with unequal field amplitudes and phase differences, which determine the far field patterns of the dimer. The asymmetric states have carrier densities with opposite signs $Z_{1} / Z_{2}=-\rho^{2}<0$ so that the 


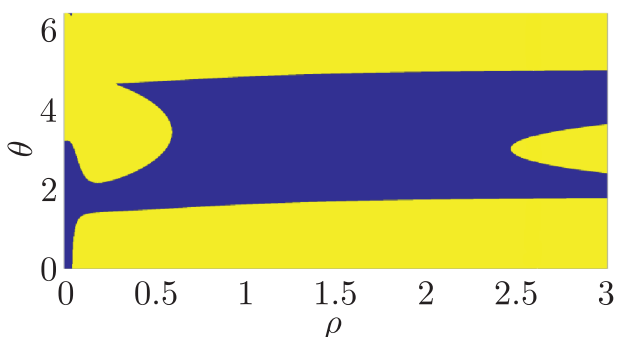

(a)

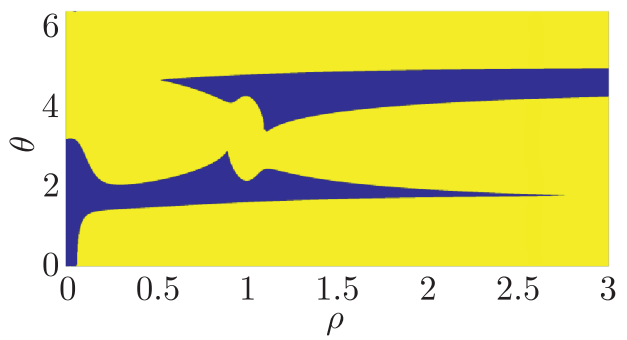

(c)

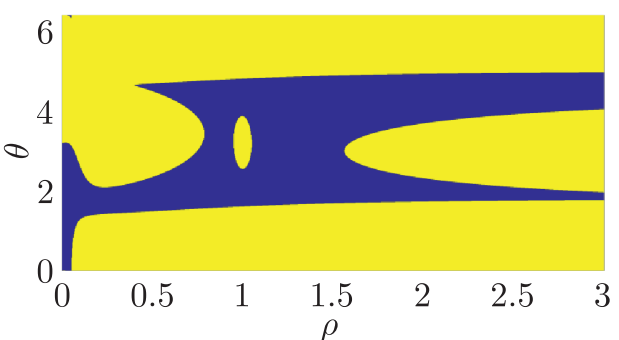

(b)

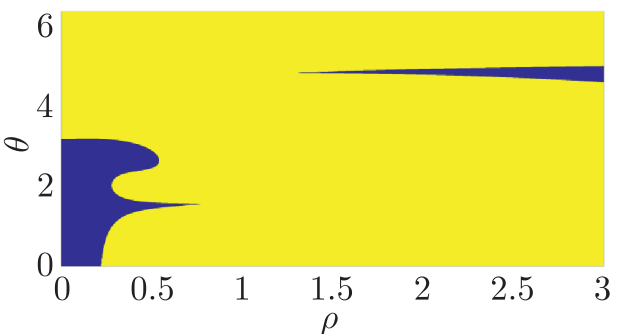

(d)

Fig. 3. Stability regions of phase-locked states of arbitrary asymmetry, characterized by the steady-state field amplitude ratio $\rho$, phase difference $\theta$ and $X_{0}=\sqrt{0.5}$. Dark blue and light yellow areas correspond to stability and instability, respectively. The parameters of the coupled lasers are $\alpha=5, T=400$ and $\log \Lambda=-2.1,-1.9,-1.7,0$ (a)-(d). The respective detuning $\Delta$ and pumping $P_{1,2}$ values are given by Eqs. (2.13)-(2.14). The stability regions are symmetric with respect to the transformation $\rho \rightarrow 1 / \rho$ and $\theta \rightarrow 2 \pi-\theta$. The topology and the extent of the stability region depend crucially on the coupling coefficient $\Lambda$.

electric fields of the lasers experience gain and loss. For $\rho=1$ we have equal gain and loss and a phase-locked state with equal field amplitudes and a phase difference given by Eq. (2.13) as $\sin \theta=-\Delta / 2 \alpha \Lambda$. When we cross the boundaries of the stability regions, the system undergoes Hopf bifurcations giving rise to stable limit cycles characterized by asymmetric synchronized oscillations of the electric fields that can have different mean values and amplitudes of oscillation.

Characteristic cases for the time evolution of the electric field amplitudes $X_{1,2}$ and the phase difference $\theta$ are depicted in Fig. 4 for various degrees of asymmetry. The parameters of the system correspond to those of Fig. $3 \mathrm{a}$ and the initial conditions are written in the caption, with phase difference $\theta=0.9 \pi \simeq 2.83$ and various values of $\rho$. For $\rho=0.75$ [Fig. 4a] the asymmetric phase-locked state is stable and perturbed initial conditions evolve to the stable state. As $\rho$ decreases to $\rho=0.5$ [Fig. 4b] and 0.25 [Fig. 4c], the phase-locked states become unstable and the system evolves to stable limit cycles of increasing period. Close to the center of the unstable region the system evolves to chaotic states $[\rho=0.15$, Fig. $4 \mathrm{~d}]$. Decreasing $\rho$ further results in stable limit cycles $[\rho=0.10$, Fig. 4e] and stable phase-locked states $[\rho=0.05$, Fig. $4 \mathrm{f}]$.

For the case of a given nonzero detuning and/or asymmetrically pumped lasers $P_{1}=P_{0}+\Delta P$, $P_{2}=P_{0}-\Delta P$, the equilibria of the system (2.6) cannot be analytically obtained from a given set of values $(\Delta, \Delta P)$. The respective algebraic system consists of transcendental equations and is solved by utilizing a numerical continuation algorithm, according to which we start from $\Delta=0$ and $\Delta P=0$ corresponding to the symmetric case of the two known equilibria $(\theta=0, \pi)$. For each one of them, we increase $\Delta$ and/or $\Delta P$ in small steps; in each step the solution of the previous step is used as an initial guess for the iterative procedure (Newton-Raphson method) that provides the solution. 


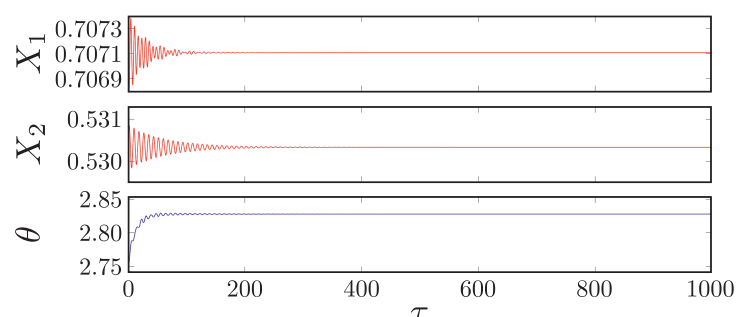

(a)

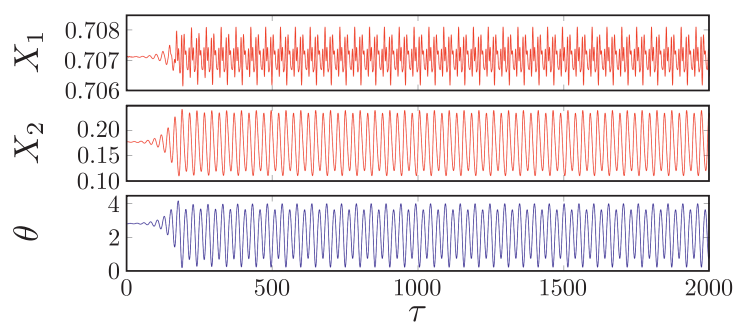

(c)

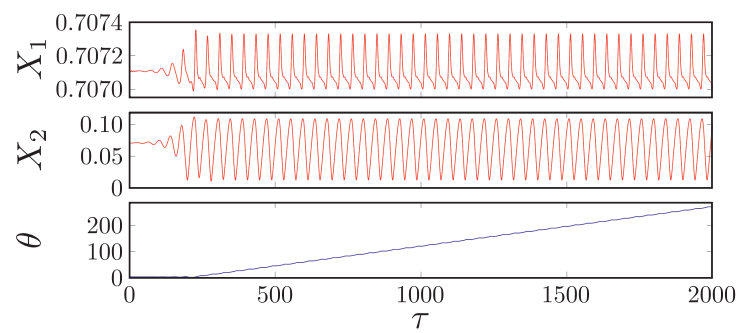

(e)

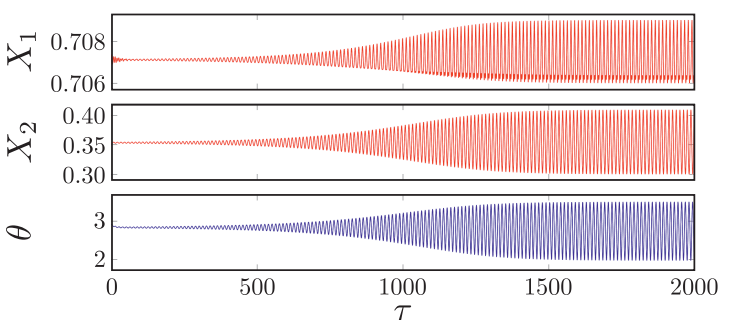

(b)

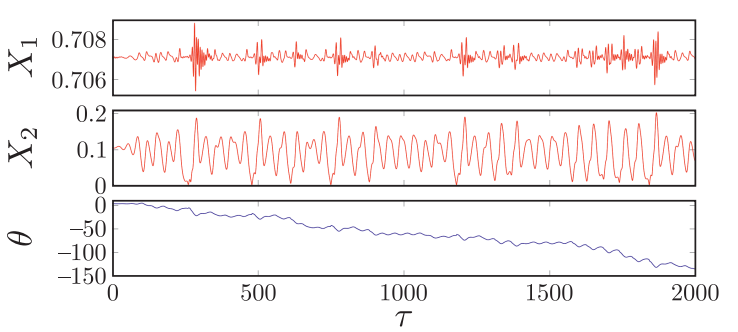

(d)

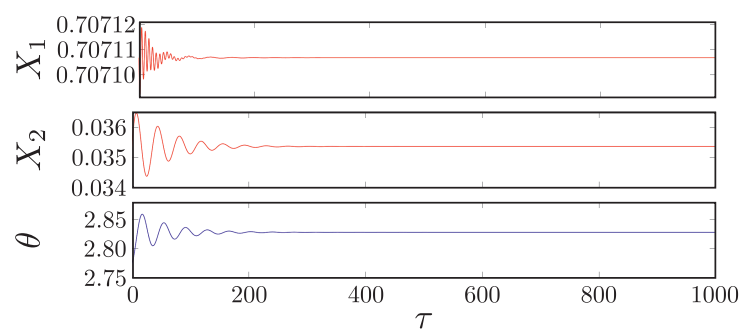

(f)

Fig. 4. Time evolution of the electric field amplitudes and phase difference for $X_{0}=\sqrt{5}, \alpha=5, T=400$, $\log \Lambda=2.1$. The initial conditions correspond to asymmetric phase-locked states with $\theta=0.9 \pi \simeq 2.83$ and $\rho=0.75(\mathrm{a}), 0.50$ (b), 0.25 (c), 0.15 (d), 0.10 (e), 0.05 (f), perturbed by random noise. Cases of stable phase-locked states are shown in (a), (f). In the case of unstable phase-locked states the system evolves either to stable limit cycles, as in (b), (c), (e), or to chaotic states, as in (d).

For the case of zero detuning $\Delta=0$, the domain of existence of stable phase-locked states in the $(\Lambda, \Delta P)$ parameter space is shown in Fig. 5a. For $\Delta P=0$ the results are similar to the case considered in [29], with the in-phase state being stable for large $\Lambda$ and the out-of-phase being stable for small values of $\Lambda$. As the pumping difference increases, the stable in-phase state extends over quite a small range of $\Delta P$, whereas the out-of-phase state extends almost in the entire range of $\Delta P$. In both cases, as $\Delta P$ increases from zero the phase difference is slightly differentiated from the values $\theta=0, \pi$ for $\Delta P=0$.

Surprisingly, another region of stable phase-locked states appears in the strong coupling regime (large $\Lambda$ ) above a threshold of pumping difference $\Delta P$. This is an out-of-phase state with phase difference close to $\pi$ that appears for values of $\Delta P$ for which no stable in-phase state exists. In fact, this stable state exists for a large part of the parameter space and extends to values $\Delta P=P_{0}$ for which only one of the lasers is pumped above threshold $\left(P_{1}=1, P_{2}=0\right)$. This area of stability, extending from intermediate to high values of coupling, is enabled by the asymmetric pumping and indicates its stabilizing effect. Non-zero detuning $(\Delta \neq 0)$ between the two lasers strongly affects the existence of a stable out-of-phase state in the weak coupling regime, as shown in Figs. 5b, 5c, for $\Delta=0.05,0.1$, respectively. 


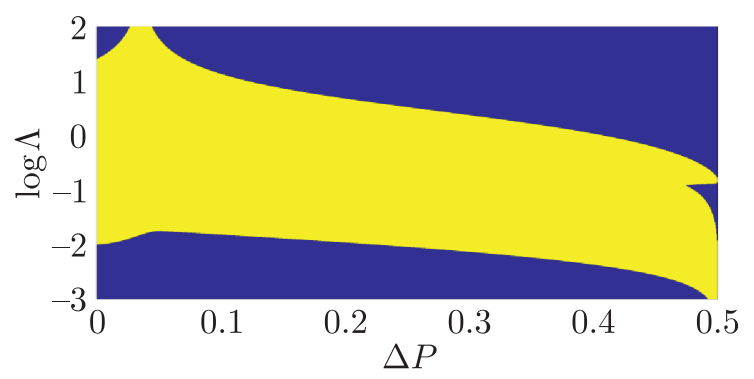

(a)

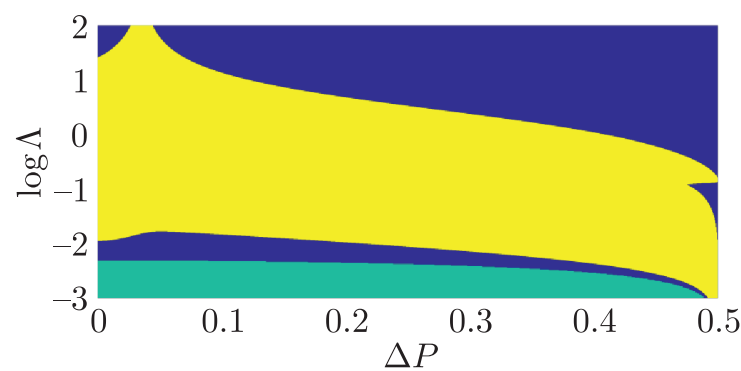

(b)

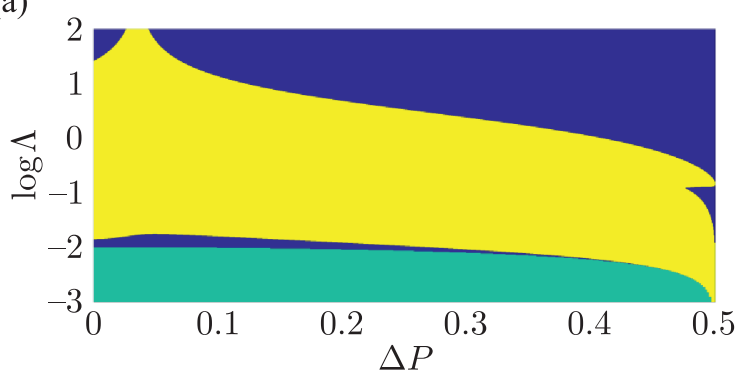

(c)

Fig. 5. Existence and stability regions of phase-locked states in the $(\Lambda, \Delta P)$ parameter space for $\alpha=5$ and $T=400$ when $P_{0}=0.5$. Dark blue and light yellow areas correspond to stability and instability, respectively. The green area corresponds to nonexistence of a phase-locked state due to nonzero detuning. (a) $\Delta=0$, (b) $\Delta=0.05$, (c) $\Delta=0.1$.

\section{Eigenvalue spectrum: exceptional points and Hopf Bifurcations}

The eigenvalues of the phase-locked states as well as the trivial zero state play a crucial role in the dynamical behavior of the system determined from the existence of Hopf bifurcation and exceptional points.

\subsection{Eigenvalue spectrum of the zero state: exceptional points and stability}

It is interesting to investigate the eigenvalue spectrum of the zero state of the system (2.1) for $M=2$. Linearizing these equations around $\mathcal{E}_{i}=0, Z_{i}=P_{i} / \Omega(i=1,2)$, we obtain a linear system whose eigenvalues are given by the characteristic equation $\operatorname{det}(A-\lambda I) \operatorname{det}\left(A^{*}-\lambda I\right) \times$ $\operatorname{det}(B-\lambda I)=0$, where

$$
A=\left[\begin{array}{cc}
(1-i \alpha) P_{1} / \Omega+i \Omega_{1} & i \Lambda \\
i \Lambda & (1-i \alpha) P_{2} / \Omega+i \Omega_{2}
\end{array}\right]
$$

$B=-\Omega /(2 P) I_{2 \times 2}, I_{2 \times 2}$ is the $2 \times 2$ unit matrix and the stars denote complex conjugation. Solving the characteristic equation, we find the eigenvalues

$$
\lambda_{1,2}=\Lambda\left\{\bar{P}+i(\bar{\Omega}-\alpha \bar{P}) \pm \sqrt{[\Delta P+i(\Delta-\alpha \Delta P)]^{2}-1}\right\}
$$

with $\lambda_{3,4}=\lambda_{1,2}^{*}$ and $\lambda_{5,6}=-1 /(2 P)$, where $\bar{P}=\left(P_{1}+P_{2}\right) / 2 \Lambda, \bar{\Omega}=\left(\Omega_{1}+\Omega_{2}\right) / 2 \Lambda, \Delta P=$ $=\left(P_{1}-P_{2}\right) / 2 \Lambda, \Delta=\left(\Omega_{1}-\Omega_{2}\right) / 2 \Lambda$ and $\Omega=1$. Since $\lambda_{5,6}$ are always negative, they correspond 
to stable directions in phase space, hence the stability of the zero state and its dependence on the parameters of the system is determined by the eigenvalues $\lambda_{1,2}$.

Note that a nonzero $\alpha$ implies that both pumping and detuning determine the real and imaginary part of the spectrum. Moreover, the carrier densities introduce the pumping rates $P_{i}$, $i=1,2$ in a way that shows their importance in determining the spectral properties of the zero state. We emphasize that these effects are absent in non-Hermitian dimers described by mode equations that neglect the nonlinear coupling of the electric fields with the respective carrier densities.

The real and imaginary parts of the eigenvalues $\lambda_{1,2}$ (normalized over the coupling constant $\Lambda$ ) as functions of the detuning $\Delta$ and pumping difference $\Delta P$ are depicted in Fig. 6 . Observe that spectral transitions, where real and imaginary parts of the eigenvalues coalesce, take place along the straight line $\Delta-\alpha \Delta P=0$ at the so-called exceptional points (EP), located at $\Delta P= \pm 1$. Exceptional points occur naturally in eigenvalue problems that depend on parameters. Varying these parameters, one can generically find EP where eigenvalues become degenerate and the corresponding eigenvectors collapse, with important consequences regarding the physical behavior of the model.

This generalizes what was known for $P T$-symmetric non-Hermitian dimers, where spectral transitions occur only along the zero detuning line $\Delta=0$ [14]. For zero detuning $\Delta=0$ and $\alpha=0$, spectral transitions take place in an analogous way as in the $P T$-symmetric case. For $|\Delta P|<1$, the eigenvalues $\lambda_{1,2}$ have a common nonzero real part and opposite imaginary parts along the line $\Delta=0$, whereas for $|\Delta P|>1$, the eigenvalues have different real parts and zero imaginary parts [Fig. 6 (top)]. For nonzero $\alpha=5$ there are no spectral transitions along $\Delta=0$. However, spectral transitions do occur along the line $\Delta=\alpha \Delta P$ as shown in Fig. 6 (bottom). One essential difference with the case of $\alpha=0$ is that the imaginary parts of $\lambda_{1,2}$ are now symmetric not with respect to zero, but with respect to a nonzero value. Therefore, for the general case of nonzero $\alpha$, asymmetric pumping can control the spectral properties
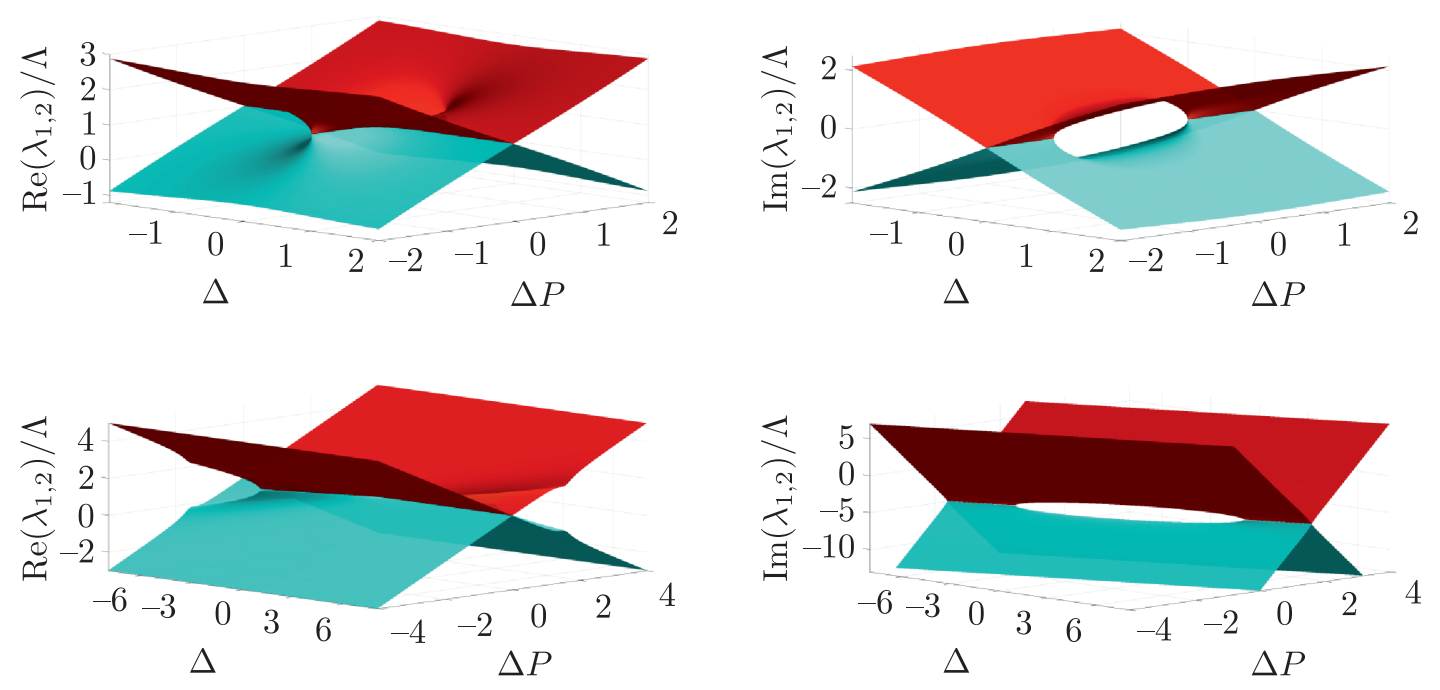

Fig. 6. Real (left) and imaginary (right) part of the normalized eigenvalues $\lambda_{1,2} / \Lambda$ of the zero state for a zero $(\alpha=0$, top) and a nonzero $(\alpha=5$, bottom) linewidth enhancement factor. Spectral transitions and exceptional points occur along the line $\Delta-\alpha \Delta P=0$. A nonzero linewidth enhancement factor raises the restriction of zero detuning $(\Delta=0)$, for the existence of exceptional points. 
of the system. Moreover, it is worth mentioning that apart from the existence of EP the linear eigenvalue spectrum of the zero state determines its stability and, as recently shown in [36], also explains phenomena related to the reversal of laser pump dependence and self-termination scenarios that can take place for varying $P_{1,2}$.

\subsection{Eigenvalue spectrum of phase-locked states: Hopf bifurcation and exceptional points}

As we realize from the above, Hopf bifurcation and exceptional points play a crucial role in laser dimer dynamics. The former corresponds to intensity peaks and the latter to side band merging [37]. To study such phenomena for asymmetric phase-locked states, we need to derive the eigenvalue spectrum of the linearized equations about these states and determine their stability and their spectral line shape. These eigenvalues are calculated from the corresponding Jacobian of the dynamical system (2.6):

$$
J=\left[\begin{array}{ll}
A_{3 \times 3} & B_{3 \times 2} \\
C_{2 \times 3} & D_{2 \times 2}
\end{array}\right]
$$

where

$$
\begin{gathered}
A_{3 \times 3}=\left[\begin{array}{ccc}
\Lambda \rho \sin \theta & -\Lambda \sin \theta & -\Lambda \rho X_{0} \cos \theta \\
\Lambda \cos \theta & -\frac{\Lambda}{\rho} \sin \theta & \Lambda X_{0} \cos \theta \\
\Lambda \frac{\rho^{2}+1}{\rho X_{0}} \cos \theta & -\Lambda \frac{\rho^{2}+1}{\rho^{2} X_{0}} \cos \theta & -\Lambda \frac{1-\rho^{2}}{\rho} \sin \theta
\end{array}\right], \\
B_{3 \times 2}=\left[\begin{array}{cc}
X_{0} & 0 \\
0 & \rho X_{0} \\
\alpha & -\alpha
\end{array}\right], \\
C_{2 \times 3}=\left[\begin{array}{ccc}
-\frac{2}{T}(1+2 \Lambda \rho \sin \theta) X_{0} & 0 & 0 \\
0 & -\frac{2}{T}\left(1-2 \frac{\Lambda}{\rho} \sin \theta\right) \rho X_{0} & 0
\end{array}\right], \\
D_{2 \times 2}=\left[\begin{array}{ccc}
-\frac{1}{T}\left(1+2 X_{0}^{2}\right) & 0 \\
0 & -\frac{1}{T}\left(1+2 \rho^{2} X_{0}^{2}\right)
\end{array}\right] .
\end{gathered}
$$

In the above expressions we have normalized the natural frequency of the dimer to $\Omega=1$, see Eq. (2.3), and replaced the scaling factor in the carrier density equations by $T$.

As is well known, stability requires that all eigenvalues have nonpositive real part. This enables us to draw stability regions in parameter space and examine what happens at their boundaries. We thus discover cases where the system undergoes a Hopf bifurcation to stable limit cycles corresponding to asymmetric synchronized oscillations of the electric fields, with generally different mean values and amplitudes [32]. EP are also found in this way, as we saw earlier in Section 3, at parameter values where two (or more) eigenvalues coalesce and can lie either in the stable or the unstable domain.

Let us focus on a system with $\alpha=5$ and carrier to photon lifetime ratio $T=400$. For zero detuning $(\Delta=0)$ and equal pumping $\left(P_{1}=P_{2}=P_{0}\right)$ we find one asymmetric phase-locked state with arbitrary amplitude ratio $(\rho)$. The phase difference and reference field amplitude are given by Eqs. (2.7) and (2.10). The stability domain of these states in parameter-solution 

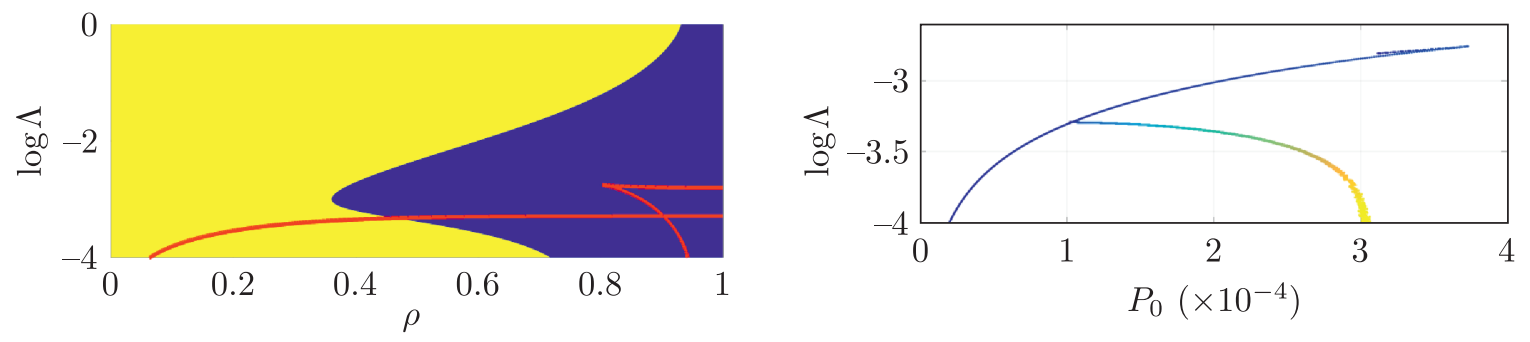

Fig. 7. Stability and location of Hopf bifurcation and exceptional points in the $(\Lambda, \rho)$ (left) and $\left(\Lambda, P_{0}\right)$ (right) space, for zero frequency detuning $(\Delta=0)$ and equal pumping rates $\left(P_{1}=P_{2}=P_{0}\right)$. (left) The Hopf bifurcation points are located at the boundary between stable (blue) and unstable (yellow) regions. Red lines depict the location of the exceptional points. (right) The color coding depicts the asymmetry of the respective phase-locked states (blue and yellow correspond to $\rho=1$ and $\rho<1$, respectively). The electric field amplitude $\left(X_{0}\right)$ is given by $(2 \cdot 10)$.
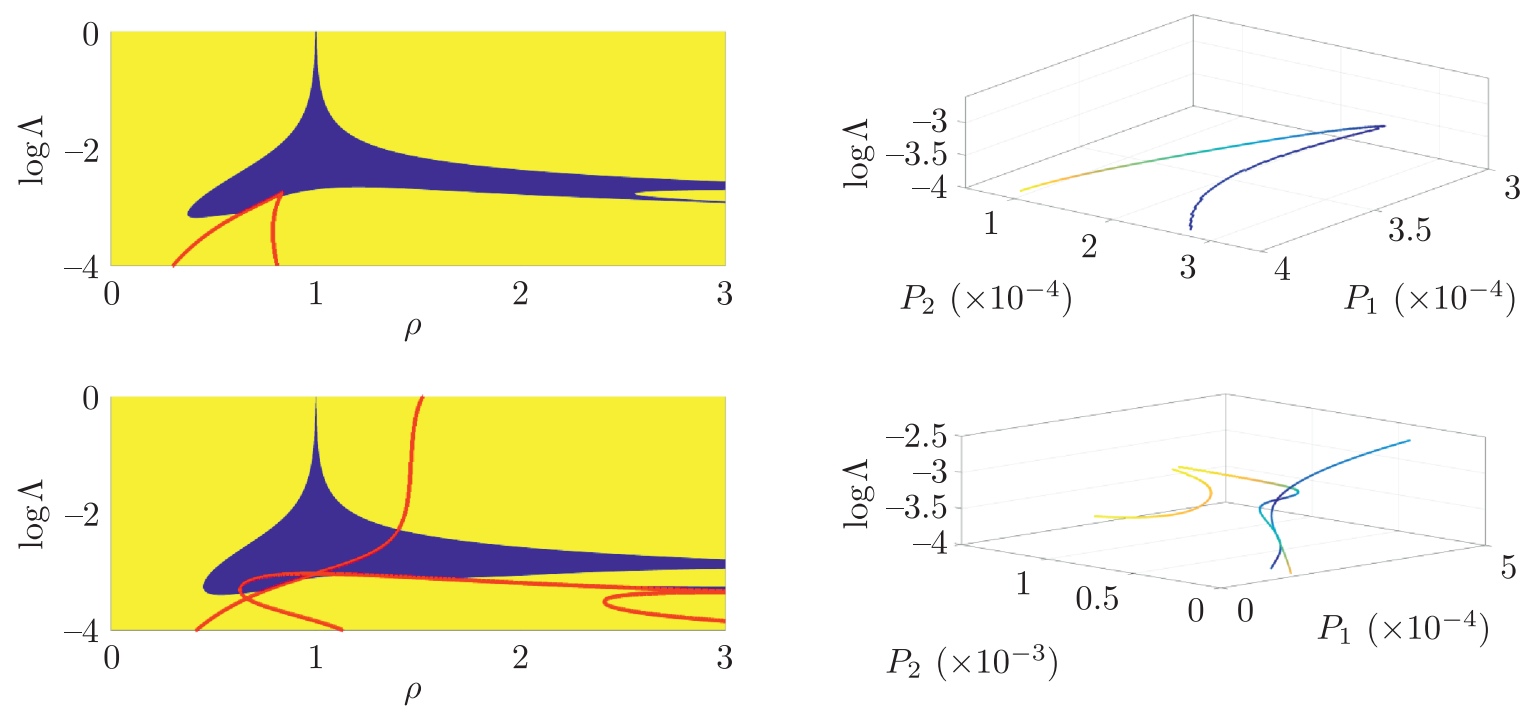

Fig. 8. Stability domains and location of Hopf bifurcation and exceptional points in the $(\Lambda, \rho)$ (left) and $\left(\Lambda, P_{1}, P_{2}\right)$ (right) space, for zero frequency detuning $(\Delta=0)$ and unequal pumping rates $\left(P_{1} \neq P_{2}\right)$. (left) The Hopf bifurcation points are located at the boundary between stable (blue) and unstable (yellow) regions. Red lines depict the location of the EP. (right) The color coding depicts the asymmetry of the respective phase-locked states (blue and yellow correspond to $\rho=1$ and $\rho<1$ or $\rho>1$, respectively). The phase difference $(\theta)$ is given by (2.7) and the electric field amplitude of the corresponding phase-locked modes is $X_{0}=10^{-1.7}$ (top) and $X_{0}=10^{-1.9}$ (bottom). Clearly, the topology of the EP lines depends crucially on the value of $X_{0}$.

space $(\Lambda, \rho)$ is depicted in Fig. 7 (left) with the Hopf bifurcation points corresponding to the boundary between the stable and the unstable region and the EP are depicted by a red line.

It is worth emphasizing that EP can be located either in the stability or in the instability region. The location of EP in the parameter space $\left(\Lambda, P_{0}\right)$ is depicted in Fig. 7 (right) with the line colormap corresponding to different values of phase-locked state asymmetry (blue and yellow correspond to $\rho=1$ and $\rho<1$, respectively). This case corresponds to the aforementioned "missed" asymmetric phase-locked state in a fully symmetric configuration examined in [29] and shows clearly the relation between the existence of EP and the inherently asymmetric dynamics of the system. 
For the case of zero detuning $(\Delta=0)$ and unequal pumping $\left(P_{1} \neq P_{2}\right)$, for every value of $\rho$ there exist two asymmetric phase-locked states with phase differences given by Eq. (2.7) for $s=0,1$, respectively. Their stability as well as the location of the Hopf bifurcation and EP are depicted in Fig. 8 (left) for different values of $X_{0}$ which is not fixed in this case. Moreover, the parameter space has an additional dimension $\left(\Lambda, P_{1}, P_{2}\right)$ and the location of the EP in this space is depicted in Fig. 8 (right). A qualitativily different topology of the EP lines is evident, including open and closed lines as well as multiple intersecting lines, depending on $X_{0}$.

\section{Spectral signatures of exceptional and Hopf bifurcation points}

In what follows we show that the existence of EP and Hopf bifurcation points result in specific spectral signatures in the spectral line shape and the modulation response of the system.

\subsection{Exceptional points and spectral line shape}

In order to study the coherence properties of a specific phase-locked state, we study the response of the linearized system in the presence of noise sources. The system under study has the form

$$
\dot{\delta \vec{X}}=\mathbf{J} \overrightarrow{\delta X}+\vec{n}
$$

where $\overrightarrow{\delta X}$ denotes small deviations from the phase-locked state $\vec{X}=\left(X_{1}, X_{2}, \theta, Z_{1}, Z_{2}\right)$, and $\vec{n}$ corresponds to amplitude, phase and carrier density fluctuations under zero-mean, deltacorrelated stochastic signals. By taking the Fourier transform of Eq. (4.1) we obtain the transfer function (matrix) of the system as

$$
\mathbf{H}(\delta \omega)=(i \delta \omega \mathbf{I}-\mathbf{J})^{-1},
$$

where $\delta \omega$ is the spectral component of the system response and $\mathbf{I}$ is the unit matrix. The power spectral density $S_{X X}$ of the output of the linear system (4.1) is then given by [38]

$$
\mathbf{S}_{\mathbf{X X}}(\delta \omega)=\mathbf{H}(\delta \omega) \mathbf{H}^{\dagger}(\delta \omega),
$$

where the $\dagger$ denotes the Hermitian conjugate of the matrix. The spectral line shape of the phase-locked states in terms of the total optical power is given by $S_{X_{1} X_{1}}+S_{X_{2} X_{2}}$ shown here in Fig. 9 (bottom row). It is clear that, in all cases, the spectral line shape and its dependence on the specific phase-locked state (as a function of $\rho$ ) follows that of the imaginary part of the eigenvalues. The observable spectral signature of the EP (vertical green dotted lines) corresponds to the emergence of side bands and the observable spectral signature of the bifurcations at zero crossings of the real part of the eigenvalues (vertical yellow dotted lines) corresponds to the appearance of points of maximum intensity.

\subsection{Hopf bifurcations and modulation response}

Let us now take advantage of our explicit knowledge of the stability domain in parameter space to modulate an asymmetric phase-locked state and investigate a number of interesting response features of our laser dimer. The small signal (linear) modulation response to a timevarying current

$$
\begin{aligned}
& P_{1}=P_{0}+\operatorname{Re}\left\{\delta P e^{i \omega t}\right\}, \\
& P_{2}=P_{0}+s \operatorname{Re}\left\{\delta P e^{i \omega t}\right\}
\end{aligned}
$$



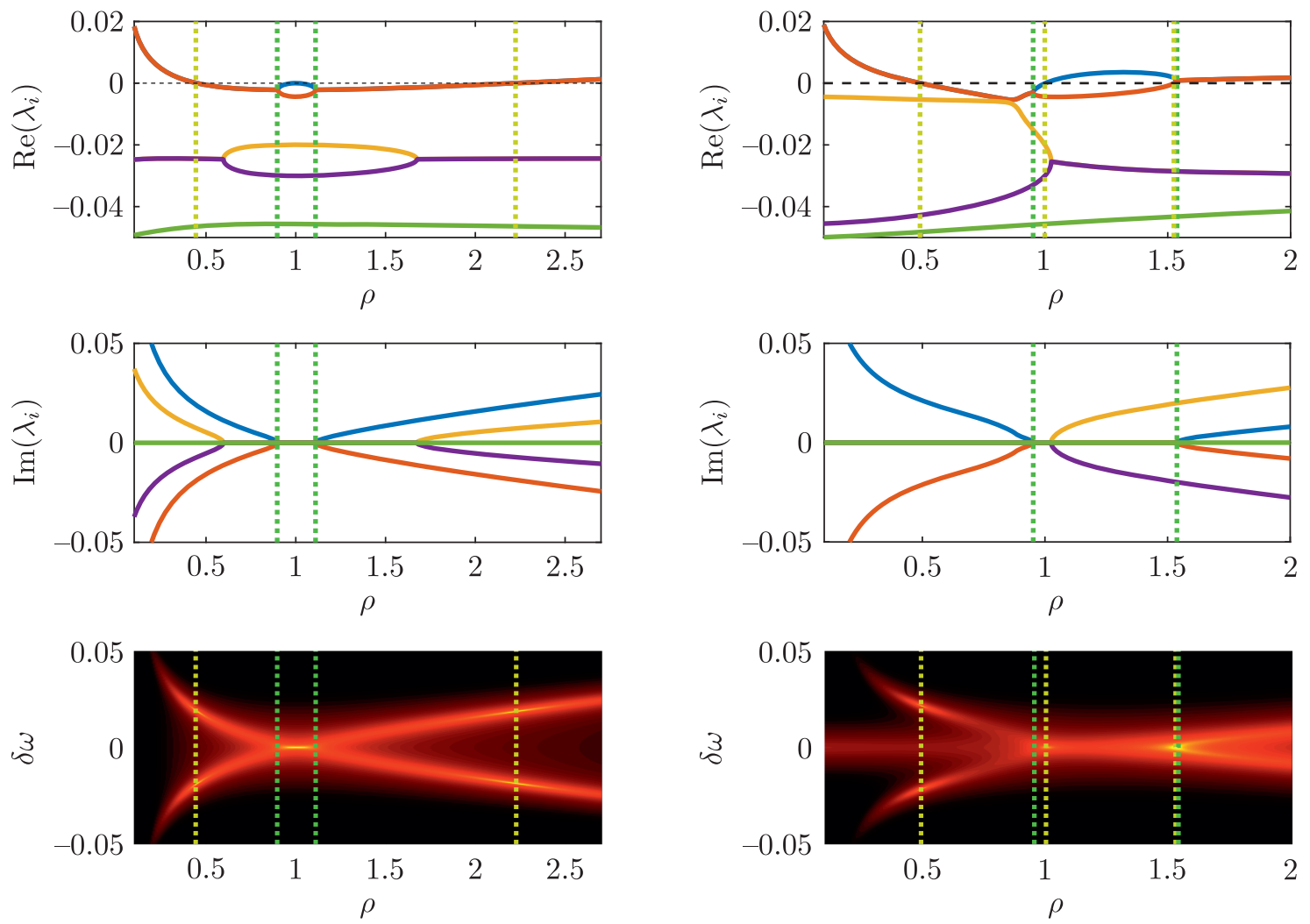

Fig. 9. Eigenvalues (top, middle) and spectral line shape (bottom) of the phase-locked states for $\alpha=5$, $\Delta=0$ and $\log \Lambda=-2$. The vertical yellow and green dotted lines correspond to zero crossings of the eigenvalue real part and exceptional points, respectively. (left) Phase-locked states under symmetric pumping $P_{1}=P_{2}\left(\theta\right.$ and $X_{0}$ are determined by $\left.\rho\right)$. The phase-locked states are stable between the vertical yellow dotted lines. (right) Phase-locked states under asymmetric pumping $P_{1} \neq P_{2}$ ( $\theta$ is determined by $\rho$ and $X_{0}=0.01$ ). The phase-locked states are stable between the first and the second (left to right) vertical yellow dotted lines. The spectral line shape follows the imaginary part of the eigenvalues. Exceptional and bifurcation points are manifested in the spectral line shape through the emergence of side bands and intensity peaks, respectively.

is calculated by linearizing the system around a stable phase-locked state, where $s= \pm 1$ corresponds in-phase and out-of-phase modulation, respectively. Defining $\vec{X} \equiv\left(X_{1}, X_{2}, \theta, Z_{1}, Z_{2}\right)$ and setting $\vec{X}=\vec{X}_{0}+\delta \vec{X}$, where $\vec{X}_{0}$ denotes the phase-locked state and $\delta \vec{X}$ is small, the frequency response of the system is obtained as follows:

$$
\delta \vec{X}=\mathbf{H}(\omega) \vec{A} \delta P
$$

with $\vec{A}=(0,0,0,1 / T, s / T)$,

$$
\mathbf{H}(\omega)=(i \omega \mathbf{I}-\mathbf{J})^{-1}
$$

the transfer matrix of the linear system and $\mathbf{J}$ its Jacobian. The amplitude response is then given by

$$
\left|\frac{\delta X_{i}}{\delta P}\right|=\frac{1}{T}\left|H_{i, 4}(\omega)+s H_{i, 5}(\omega)\right|, \quad i=1,2 .
$$

Depending on the asymmetry of the modulated phase-locked state and the coupling, we discover that the linear modulation response of our system has remarkable features of physical 


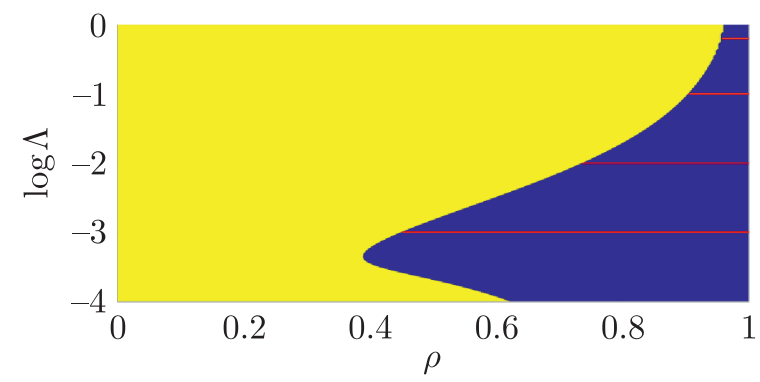

(a)

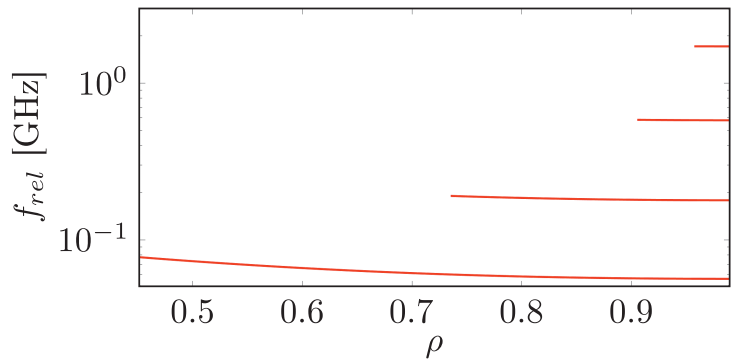

(b)

Fig. 10. (a) Stability region (blue shaded area) of symmetry-breaking phase-locked states with electric field amplitude ratio $\rho$ for varying normalized coupling coefficient $\Lambda$. The horizontal (red) lines correspond to $\Lambda=10^{-3}, 10^{-2}, 10^{-1}, 10^{-0.2}$. (b) Free running relaxation frequency $\left(f_{\text {rel }}\right)$ as a function of $\rho$ for the aforementioned values of $\Lambda$.

significance, including resonances and antiresonances at frequencies far beyond the free running relaxation frequencies of the dimer, cf. Eq. (2.2) and Fig. 10b.

For strong coupling, the stable phase-locked states are slightly asymmetric ( $\rho$ close to unity) in comparison with the weak coupling regime and the respective free running relaxation frequencies are of the order of $\mathrm{GHz}$ as shown in Fig. 10. In such cases, the in-phase modulation response has a peak close to the free running relaxation frequency which is smaller than $1 \mathrm{GHz}$, for a coupling coefficient $\Lambda=10^{-1}$ (see Fig. 10b). For the out-of-phase modulation, a sharp peak appears at a frequency that is several times larger than the free running relaxation frequency and its maximum depends weakly on the asymmetry $(\rho)$.

For even larger values of the coupling, $\Lambda=10^{-0.2}$ and out-of-phase modulation we observe the same phenomenon, but now with a resonant peak appearing at a frequency near $100 \mathrm{GHz}$, which is more than 50 times larger than the free running relaxation frequency, as shown in Fig. 11. As the stable phase-locked states for strong coupling are weakly asymmetric, with $\rho$ close to unity, the high-frequency peaks of the out-of-phase modulation response appear at frequencies that are close to the analytically known frequencies [47, 48]

$$
\Omega=2 \Lambda+\Omega_{r e l}^{2} / \Lambda \text {. }
$$

For weak coupling, e.g., $\Lambda=10^{-3}$, the stable phase-locked states can be highly asymmetric, with $\rho$ significantly different from unity, as shown in Fig. 12a with their free running relaxation frequencies being of the order of $0.1 \mathrm{GHz}$ and depending on $\rho$, as illustrated in Fig. 12b. The amplitude modulation response for a coupling coefficient $\Lambda=10^{-3}$ is shown in Figs. 12 and 13 for out-of-phase and in-phase modulation, respectively. In both cases, a smooth peak appears at the free running relaxation frequency and sharp peaks appear at a higher frequency for strongly asymmetric phase-locked modes. These modes are close to the stability boundary (Fig. 9a) where a Hopf bifurcation takes place [36].

For the case of in-phase modulation, an interesting antiresonance phenomenon appears as a sharp dip in the modulation response of the first laser, shown in Fig. 14a. The term antiresonance has been used in a variety of physical systems related to cavity QED [49], metamaterials [50] and vibration testing [51]. Interestingly, it seems to have passed unnoticed in the photonics literature of small signal modulation of semiconductor lasers. A careful review of recent work [52] and [53], however, reveals that it was evident in a number of experimental and theoretical studies [54]. 

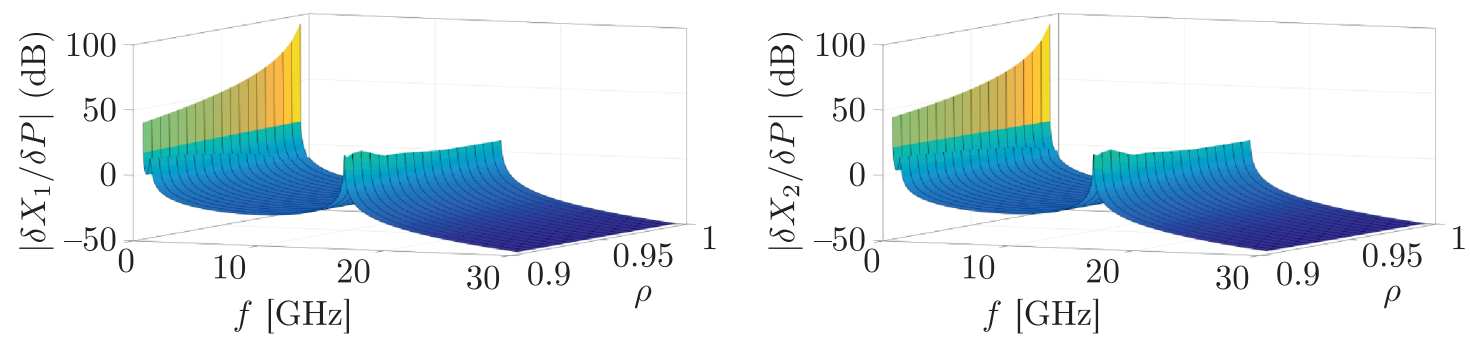

Fig. 11. Modulation response as a function of the electric field amplitude ratio $\rho$ for $\Lambda=10^{-1}$ under out-of-phase modulation. The amplitude is peaked at a much higher frequency than the free running relaxation frequency and weakly depends on $\rho$.
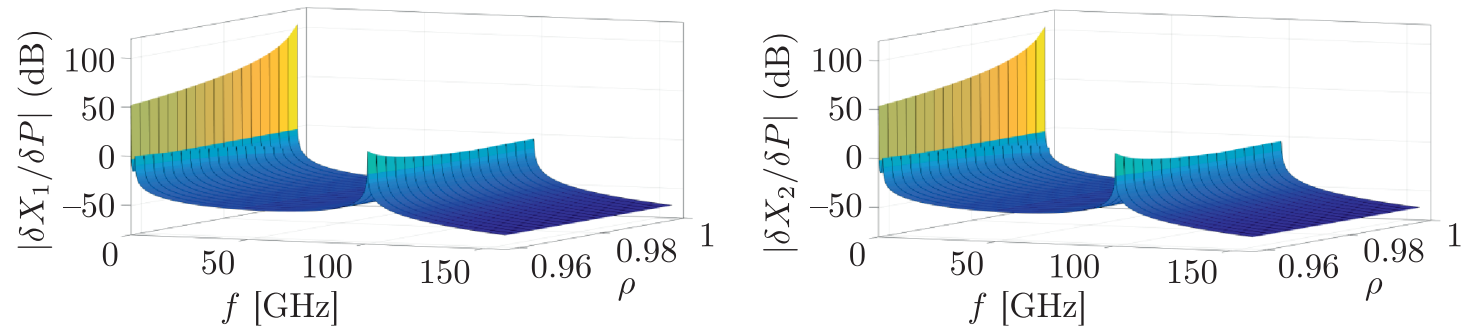

Fig. 12. Modulation response as a function of the electric field amplitude ratio $\rho$ for $\Lambda=10^{-0.2}$ under out-of-phase modulation. The amplitude is now peaked at much higher frequency (beyond $100 \mathrm{GHz}$ ) than what was found in Fig. 10.
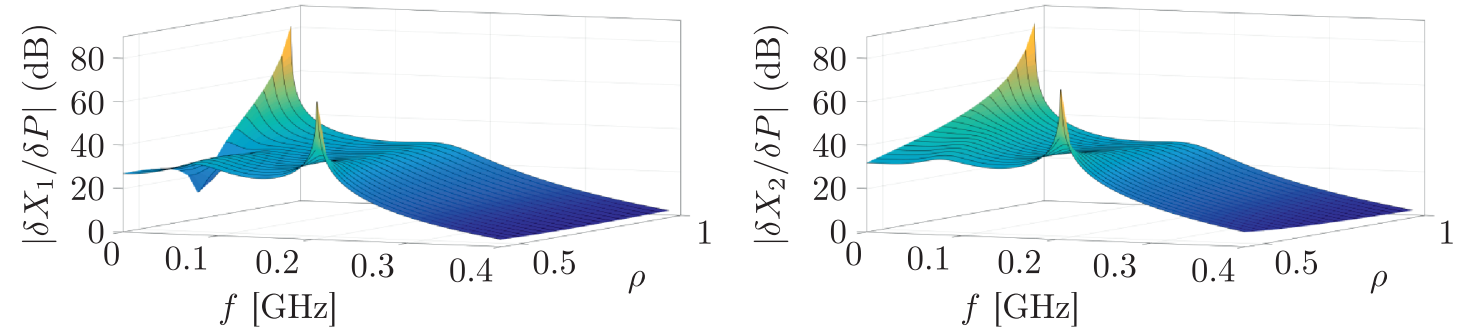

Fig. 13. Modulation response as a function of the electric field amplitude ratio $\rho$ for $\Lambda=10^{-3}$ under out-of-phase modulation. Sharp resonances appear for strongly asymmetric phase-locked states, close to Hopf bifurcation points. The resonances appear at frequencies above the free running relaxation frequency shown as a smoother peak in the modulation response.
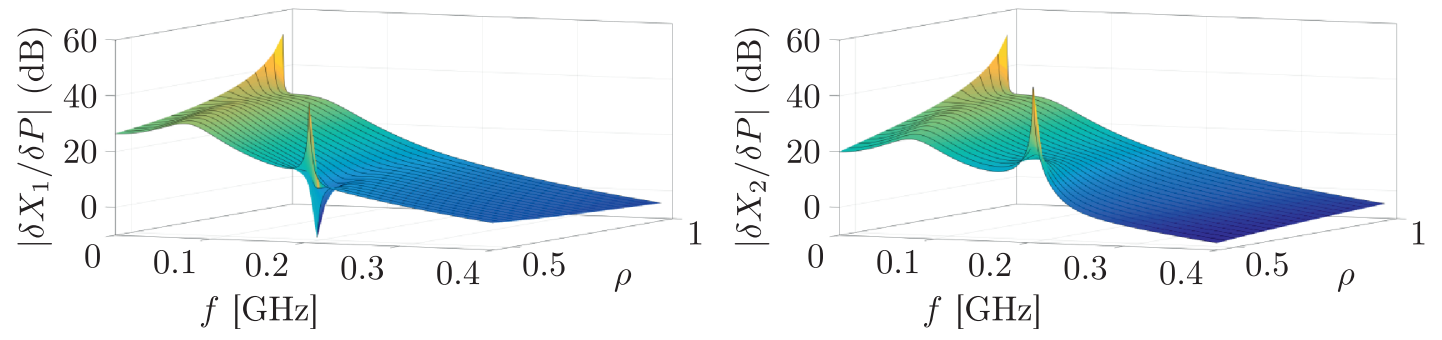

Fig. 14. Modulation response as a function of the electric field amplitude ratio $\rho$ for $\Lambda=10^{-3}$ under in-phase modulation. Sharp resonances appear in both cases. Apart from sharp resonances, antiresonances appear at the amplitude response of the first laser for strongly asymmetric phase-locked states, close to Hopf bifurcation points. Both resonances and antiresonances appear at frequencies above the free running relaxation frequency shown as a smoother peak in the modulation response. 


\section{New oscillatory phenomena in arrays of laser dimers}

We now turn to the study of the dynamics of a dimer array whose fundamental variables are the slowly varying complex amplitudes $\mathcal{E}_{j}$ of the normalized electric fields of the lasers and the corresponding population inversions as $N_{j}$, see (2.1). Each dimer consists of two lasers labeled 1 and 2 , whence the rate equations describing this system are

$$
\begin{aligned}
\frac{d \mathcal{E}_{j}^{1}}{d t} & =(1-i \alpha) N_{j}^{1} \mathcal{E}_{j}^{1}+i \eta_{i n} \mathcal{E}_{j}^{2}+i \eta_{e x} \mathcal{E}_{j-1}^{2}+i \omega_{j}^{1} \mathcal{E}_{j}^{1}, \\
\frac{d \mathcal{E}_{j}^{2}}{d t} & =(1-i \alpha) N_{j}^{2} \mathcal{E}_{j}^{2}+i \eta_{i n} \mathcal{E}_{j}^{1}+i \eta_{e x} \mathcal{E}_{j+1}^{1}+i \omega_{j}^{2} \mathcal{E}_{j}^{2}, \\
T \frac{d N_{j}^{1}}{d t} & =P_{j}^{1}-N_{j}^{1}-\left(1+2 N_{j}^{1}\right)\left|\mathcal{E}_{j}^{1}\right|^{2}, \\
T \frac{d N_{j}^{2}}{d t} & =P_{j}^{2}-N_{j}^{2}-\left(1+2 N_{j}^{2}\right)\left|\mathcal{E}_{j}^{2}\right|^{2},
\end{aligned}
$$

where $1 \leqslant j \leqslant M$ with $M$ the number of dimers, see also (2.1). $\eta_{i n}$ is the coupling between lasers 1 and 2 in each dimer, while $\eta_{e x}$ denotes the coupling between neighboring dimers, see Fig. 15. The dimensionless time $t$ and the population inversion decay time $T$ are measured in units of the field decay time. The linewidth enhancement factor is $\alpha$ and $P_{j}^{1,2}$ are the pumping coefficients. The normalized angular frequency $\omega_{j}$ measures the detuning of laser $j$ from a common reference.

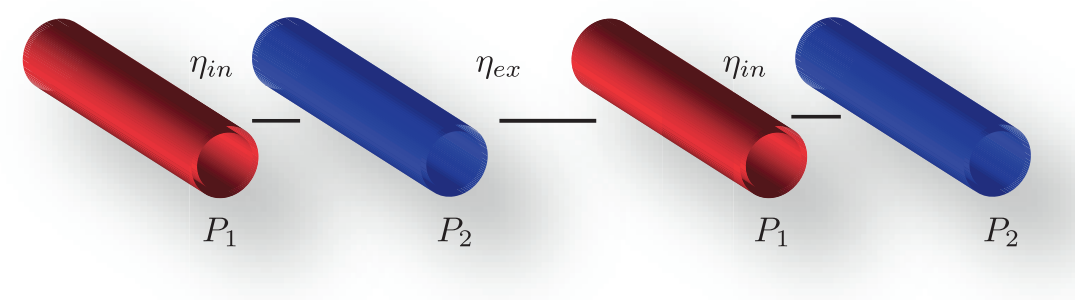

Fig. 15. Pictorial representation of part of an array consisting of two dimers. The coupling constant within the lasers of each dimer is denoted by $\eta_{i n}$, while the one between the dimers is $\eta_{e x} . P_{1}$ and $P_{2}$ denote the pumping rates in the left and right laser of each dimer, respectively.

In the case of a single dimer, equal pumping rates and in the absence of detuning the fixed point of the dimer is stable under the condition [29]:

$$
\eta<\frac{1+2 P}{2 \alpha T}
$$

Choosing as before $\alpha=5, T=400$, we find that with $P=1.5$ the phase-locked state destabilizes and leads to a limit cycle at $\eta=0.001$. Solving the equations of motion for two dimers with $P^{1}=P^{2}=1.5$, and $\eta_{i n}=\eta_{e x}=\eta$, we find that their electric fields oscillate with nearly equally large electric amplitudes, for $0.001 \lesssim \eta \lesssim 0.004$ as shown in Fig. 16 . In fact, if we couple two dimers, with $\eta_{i n}=0.0025$, we find that the oscillatory dynamics of each dimer persists for quite a large interval of $\eta_{e x}$, see Fig. 17. 

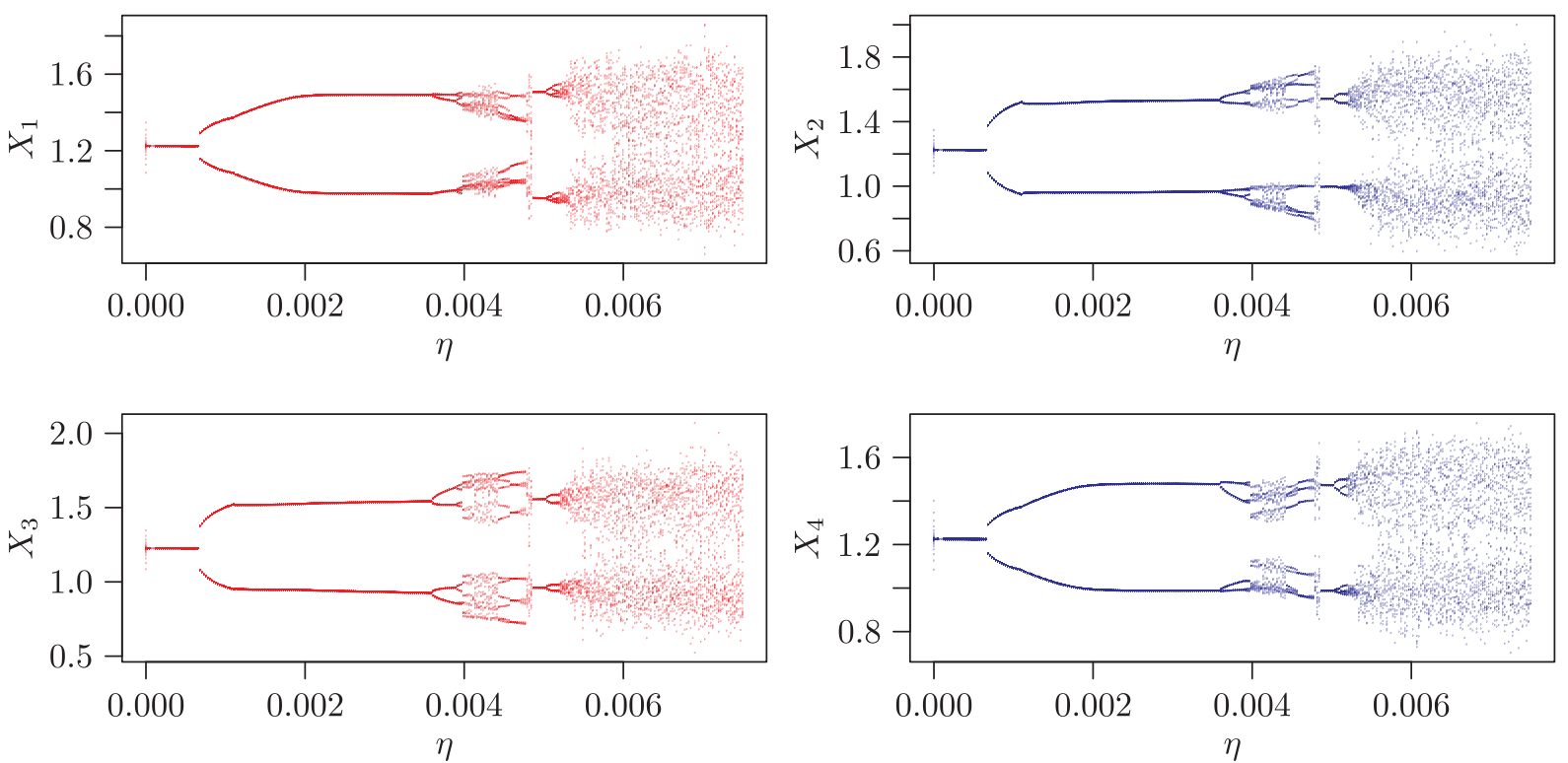

Fig. 16. Bifurcation diagram of the maxima and minima of the electric field amplitudes for two coupled symmetric dimers with $\left(P_{1}, P_{2}\right)=(1.5,1.5)$. The upper two figures correspond to dimer 1 and the lower two to dimer 2. Here we vary $\eta_{i n}=\eta_{e x}=\eta$, with $h_{\text {Hopf }}=\left(1+2 P_{0}\right) /(2 T \alpha)$ at the Hopf bifurcation point.
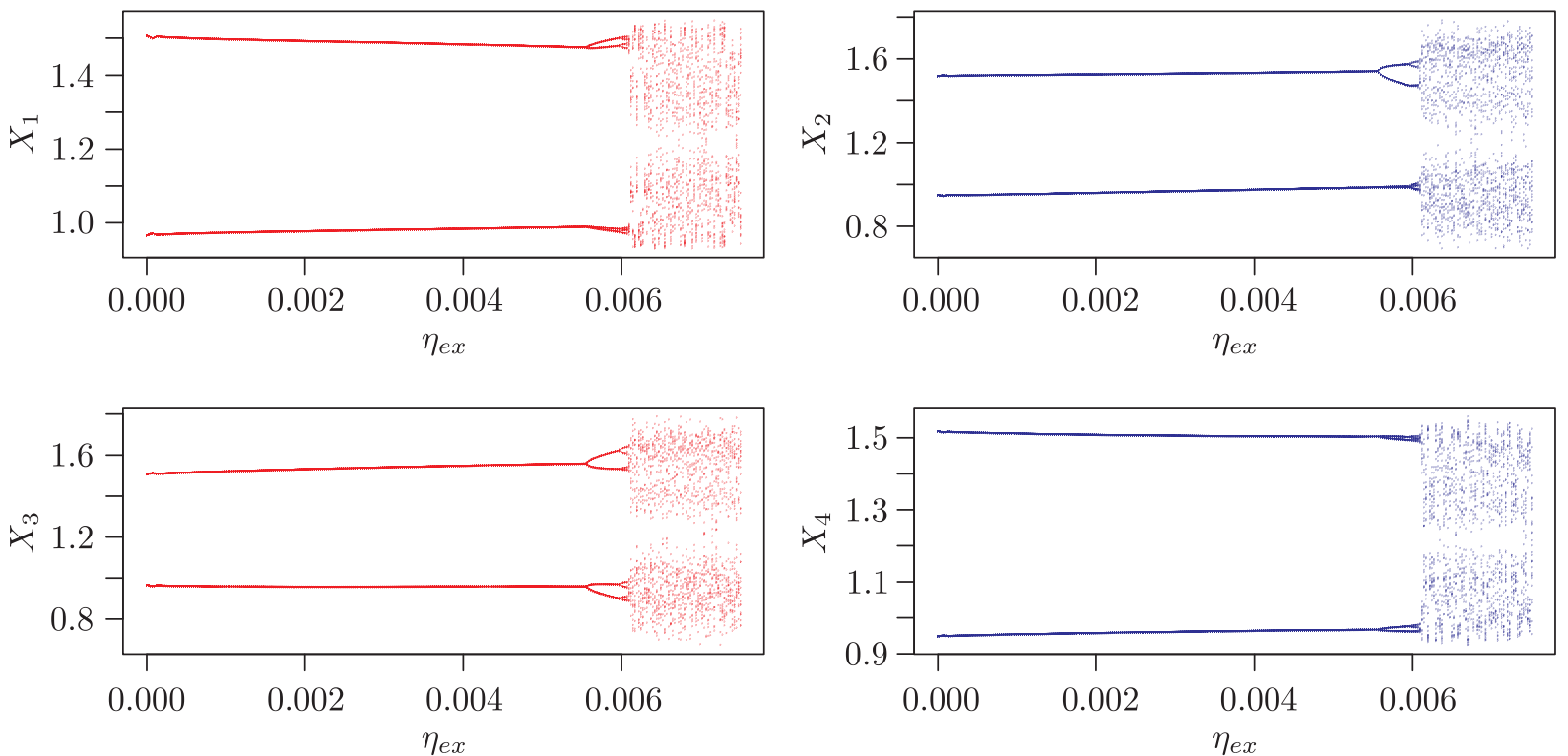

Fig. 17. Bifurcation diagram of the maxima and minima of the electric field amplitudes, as in Fig. 16. Here we vary $\eta_{e x}$ for fixed $=\eta_{i n}=0.0025$ and $\left(P_{1}, P_{2}\right)=(1.5,1.5)$.

In Fig. 18 we see that under the same setting as in the previous figures, but with slightly larger internal coupling $\eta_{i n}=0.0042$ the oscillations develop through period doubling a chaotic regime, which, however, is small and leads again to regular oscillations for higher values of $\eta_{e x}$. 

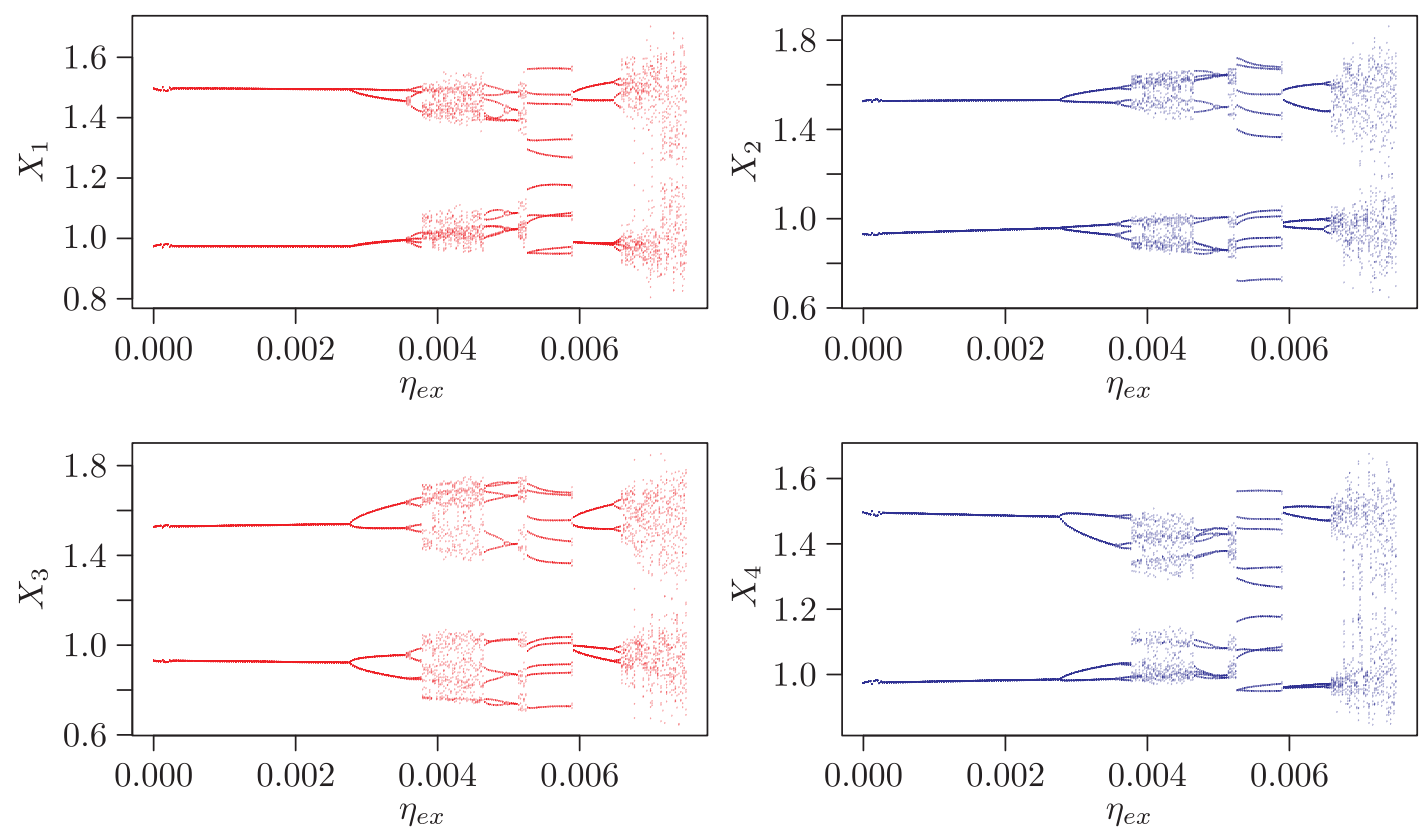

Fig. 18. Bifurcation diagram of the maxima and minima of the electric field amplitudes, as in Fig. 16. Here we vary $\eta_{e x}$ for fixed $=\eta_{i n}=0.0042$ and observe that there often exist regions of restabilization of the oscillations beyond the regimes of chaos.

\subsection{Asymmetric pumping rates and nonzero detuning}

Let us now make the lasers asymmetric by imposing different pumping rates on the dimers as follows: We set $\left(P_{1}, P_{2}\right)=(1.5,0.8)$ and vary at first both the internal and external coupling constants, taking $\eta_{e x}=\eta_{i n}$ in Fig. 19. As we see, comparing with Fig. 18, the regions of nearly equal amplitude oscillations (NEAO) have decreased somewhat, but, more importantly, the first laser in each of the dimers executes much smaller oscillations close to the (unstable) phase-locked state, which we refer to as SOPLS.

Next, we consider 3 symmetric dimers in Fig. 20 and vary $\eta_{e x}$ keeping $=\eta_{i n}=0.0025$. Again we see that all the lasers display NEAO up to $\eta_{e x} \simeq 0.005$. In fact, if we make this system asymmetric by making the detuning different from zero, i.e., $\Delta=2 \cdot 10^{-3}$ with $\left(\omega_{1}, \omega_{2}\right)=$ $=(1.001,0.999)$, we find that the electric field oscillations are very similar as in the zero detuning case.

Finally, let us consider the case of 30 dimers and take again the symmetric case of $\left(P_{1}, P_{2}\right)=$ $=(1.5,1.5)$ for each dimer with internal coupling $\eta_{i n}=0.0025$. Plotting the long-time dynamics of the first dimer (top row), the 15th (middle row) and the 30th (bottom row) in Fig. 21, we observe quite a wide regime of stable large-amplitude oscillations extending even slightly above $\eta_{e x}=0.005$. If we repeat the experiment, however, for the asymmetric case $\left(P_{1}, P_{2}\right)=(1.5,0.8)$, we find, as we saw in Fig. 19, that one of the lasers in each dimer remains close to the phaselocked state, while the other executes large oscillations, albeit somewhat more chaotically than in the symmetric case.

\subsection{Different choices of pumping rates}

There are, of course, more complex forms of asymmetry that one can impose on the dimers and study their effect on the dynamics. 

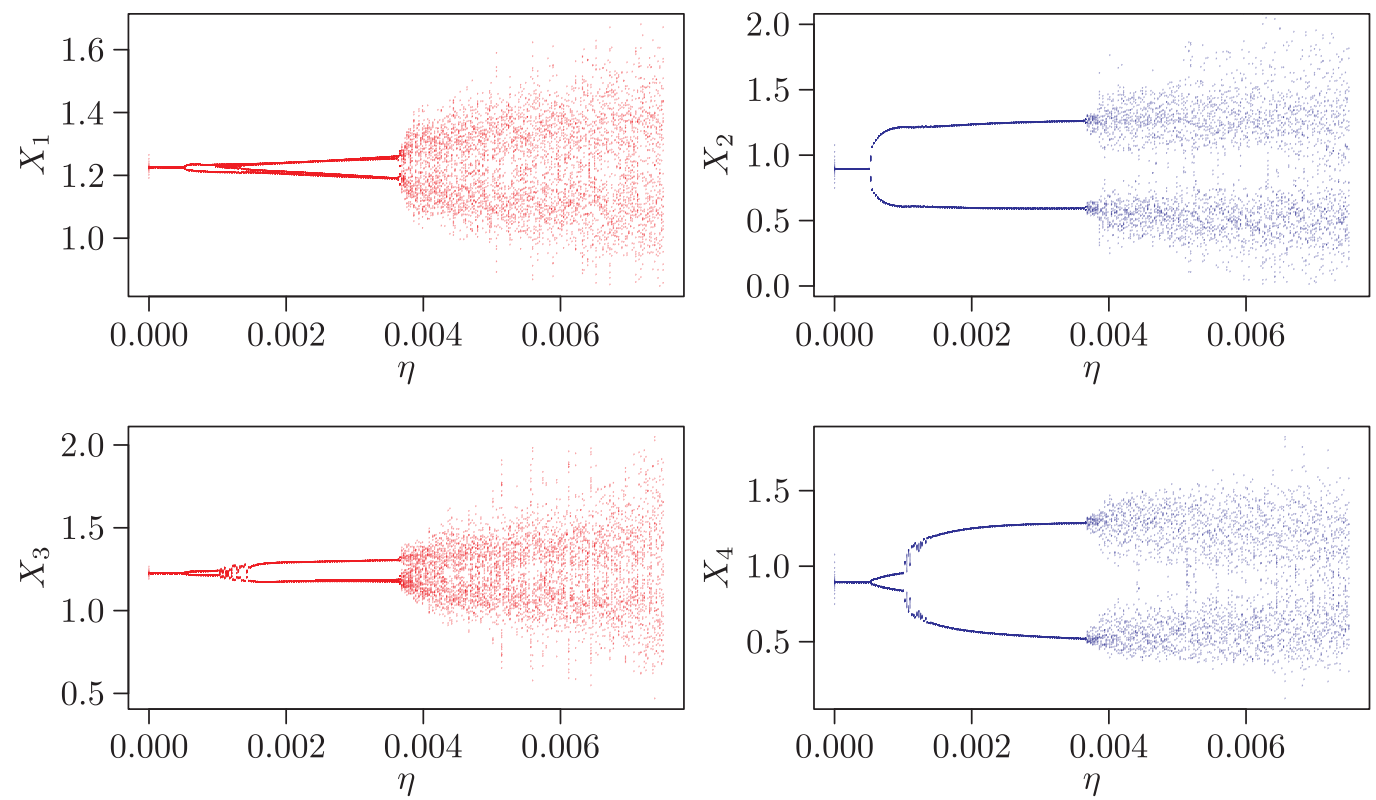

Fig. 19. Bifurcation diagram of the maxima and minima of the electric field amplitudes, for asymmetric pumping rates $\left(P_{1}, P_{2}\right)=(1.5,0.8)$. Here we vary $\eta_{e x}=\eta_{i n}=\eta$ and observe that one of the lasers in each dimer remains close to the fixed state, while the other executes large oscillations.
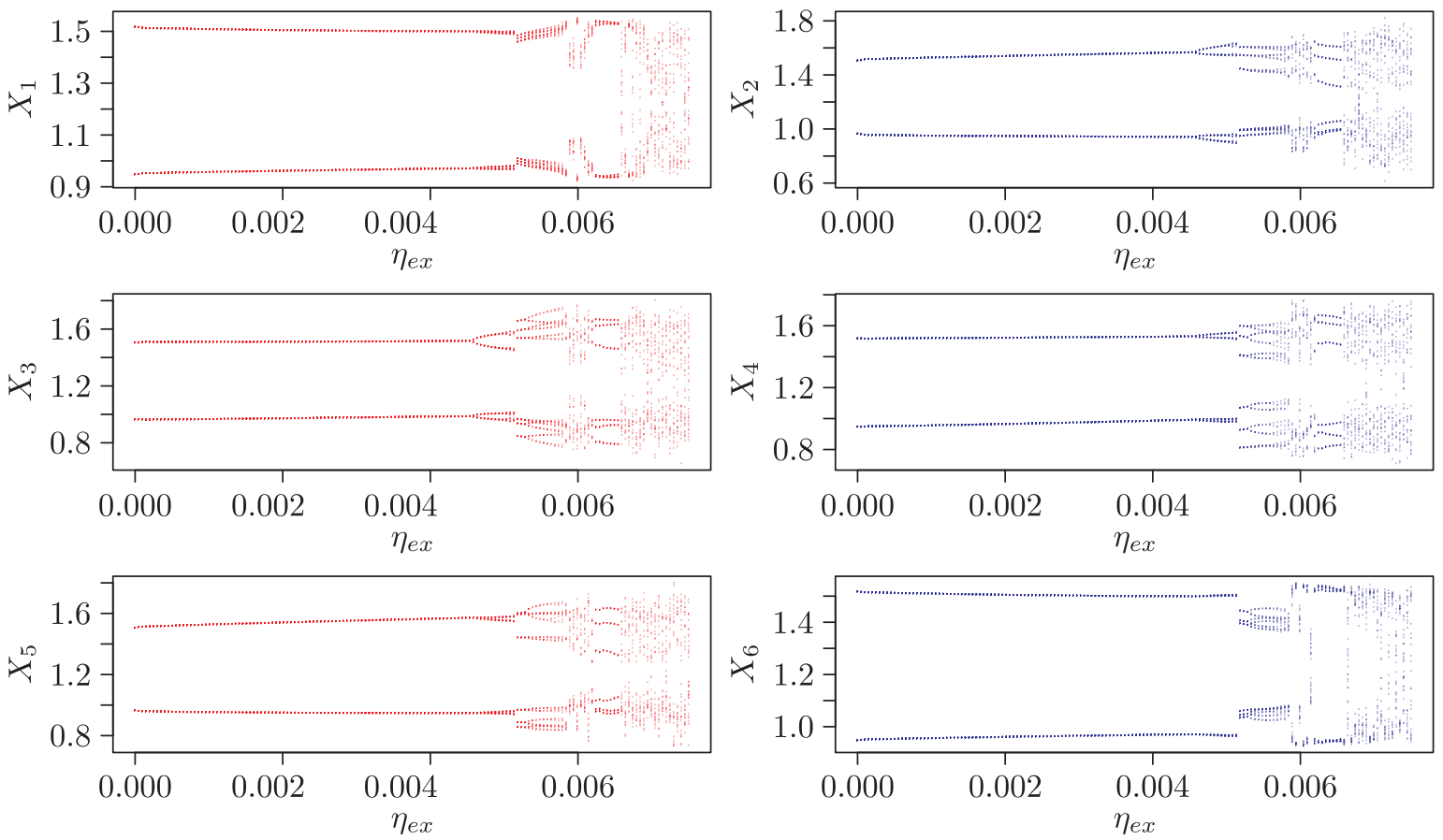

Fig. 20. Bifurcation diagram of the maxima and minima of the electric field amplitudes for 3 coupled dimers. Varying $\eta_{e x}$ for fixed $\left(P_{1}, P_{2}\right)=(1.5,1.5)$ and internal coupling $\eta_{i n}=0.0025$, we find that all lasers execute NEAO. Detuning is zero with $\omega_{j}=0, j=1, \ldots, 6$. 

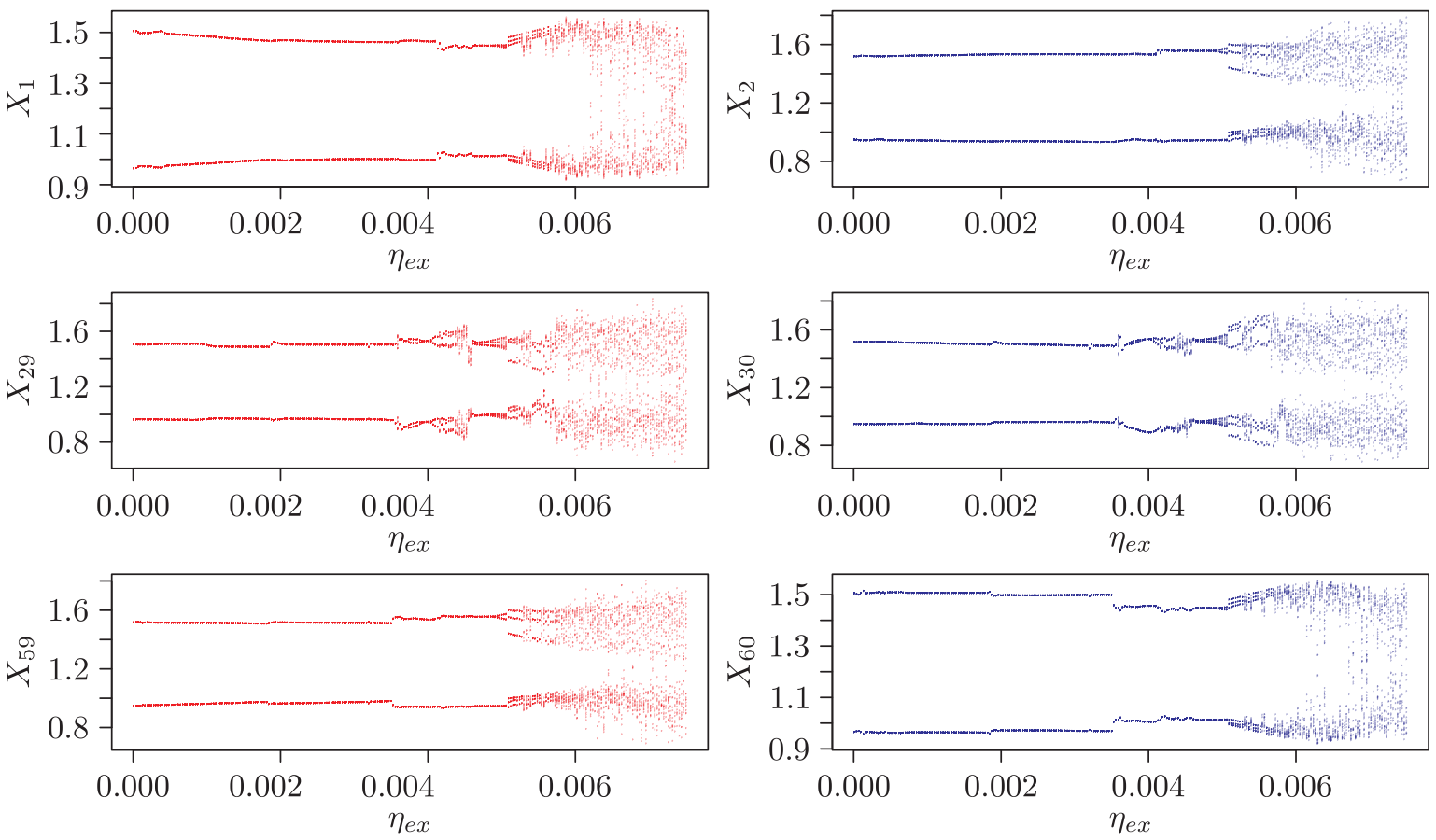

Fig. 21. Bifurcation diagram of the maxima and minima of the electric field amplitudes for 30 coupled dimers. Here we present the amplitudes of the first (top row), the 15th (middle row) and the last dimer (bottom row). Varying $\eta_{\text {ex }}$ for fixed $\left(P_{1}, P_{2}\right)=(1.5,1.5)$ and internal coupling $\eta_{\text {in }}=0.0025$. Zero detuning with $\omega_{j}=0, j=1, \ldots, 60$.

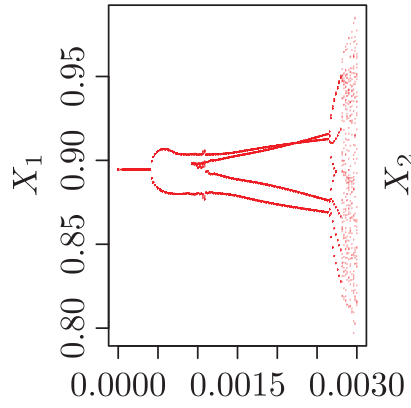

$\eta$

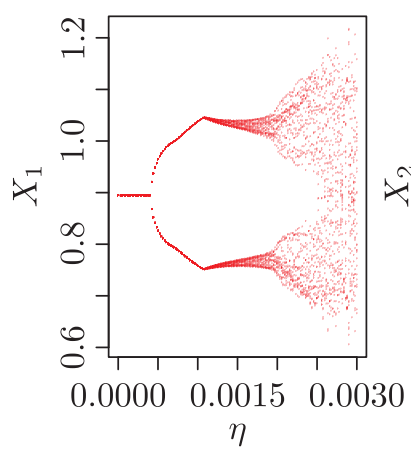

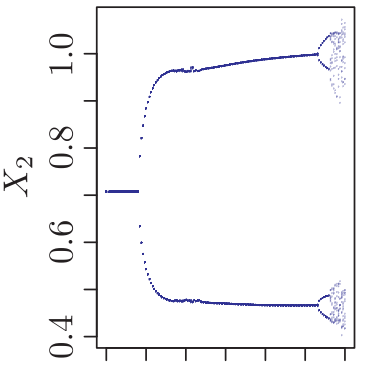

$\begin{array}{lll}0.0000 & 0.0015 & 0.0030\end{array}$

$\eta$

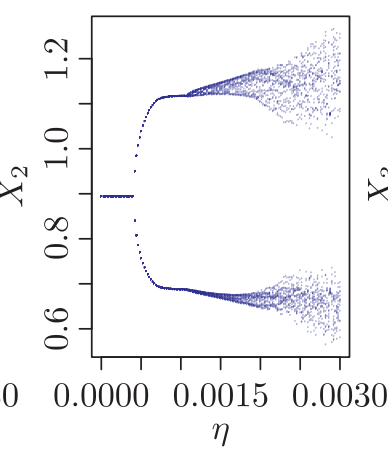

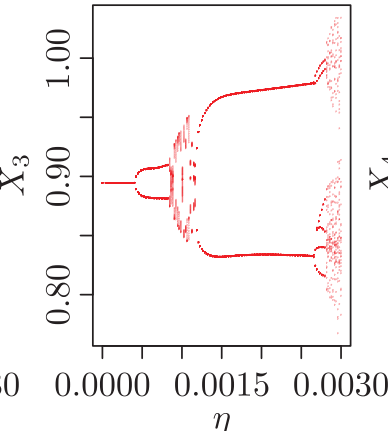

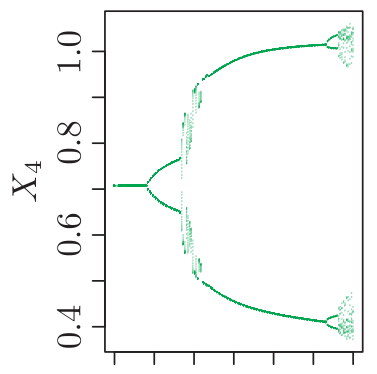

$0.0000 \quad 0.0015 \quad 0.0030$

$\eta$
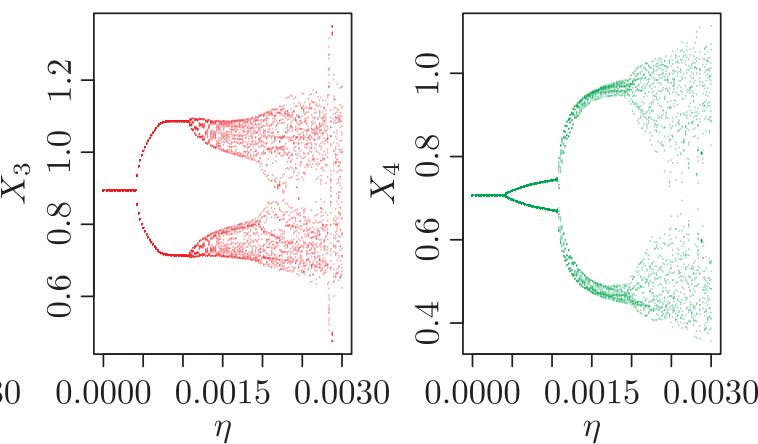

Fig. 22. Bifurcation diagram of the maxima and minima of the electric field amplitudes for two coupled dimers, as $\eta_{\text {in }}=\eta_{e x}=\eta$ varies. Top row dimer: $P_{1}=P_{3}=0.8, P_{4}=0.5$ and $P_{2}=0.5$. Bottom row dimer: $P_{1}=P_{3}=0.8, P_{4}=0.5$ and $P_{2}=0.8$. 
In Fig. 22 we present the bifurcation diagrams for two dimers, with $P_{1}=P_{3}=0.8, P_{4}=0.5$ and $P_{2}=0.5$ for the top row dimer, and $P_{2}=0.8$ for the bottom dimer, and equal coupling strengths $\eta_{i n}=\eta_{e x}=\eta$. Note that for low values of $\eta$ the dimers are synchronized at phaselocked states, but at approximately $\eta=0.0005$ the system undergoes a Hopf bifurcation, giving rise to limit cycles. The main difference between the two cases is that with $P_{2}=0.8$ we observe a quick transition to chaotic motion at about $\eta=0.001$, whereas with $P_{2}=0.5$ the system exhibits either SOPLS or NEAO dynamics before a period doubling cascade around $\eta=0.0025$.

We finally mention that the above results were checked and found to be quite robust under small additive noise, as well as periodic boundary conditions.

\section{Conclusions and future outlook}

In the present paper, we first reviewed a number of key results of our group over the past 3 years, concerning the dynamics of coupled semiconductor lasers, with the purpose of constructing photonic arrays that would be useful in optical communications, sensing and imaging. In particular, our main goal is to look for appropriate configurations that would allow us to control the operation and tunability of these arrays so as to be able to obtain desirable field patterns.

Thus, we first outlined our studies on a single pair of coupled semiconductor lasers, called a dimer, which is known to exhibit stationary states, Hopf bifurcations, limit cycles and transitions to chaos that can be controlled by varying the pumping rates $P_{1}, P_{2}$ entering the carrier density equations of the two lasers. We thus showed that there exist stable asymmetric phaselocked states with unequal field amplitudes and phase differences determining the dimer's far field patterns, enabling it to act as a unit of large controllable photonic structures. We also showed that, remarkably, stable asymmetric modes exist even in the symmetric case where the two lasers are homogeneously pumped. These modes bifurcate to stable limit cycles through Hopf bifurcations, while nonzero detuning and asymmetric pumping can lead to phase-locked modes with arbitrary power, amplitude ratio and phase difference. We emphasized that such deviations from $P T$-symmetry can lead to asymmetric phase-locked states with arbitrary field amplitude ratio, which is especially desirable for applications.

The eigenvalue spectra of both the trivial zero state and the asymmetric phase-locked states were systematically analyzed and the existence of exceptional points (EP) as well as Hopf bifurcation points was shown to be enabled in extended regions of the parameter space due to asymmetry. For a long time, EP in parameter space had been considered only in connection with PT-symmetry. In our work, we demonstrated that EP also exist under asymmetric configurations, thus allowing us to vary coupling, frequency detuning and pumping rates to achieve the desired experimental specifications. The EP were shown to correspond to specific spectral signatures on the spectral line shape of the system. Moreover, we studied the response of a single dimer to small signal modulations, when one varies the asymmetry of certain stable phase-locked states. This has led us to the discovery of frequency responses, with remarkable properties such as sharp resonances and antiresonances, at frequencies that are sometimes orders of magnitude higher than the dimer's relaxation frequency.

Finally, we turned to some preliminary new results concerning the dynamics of arrays of coupled dimers under nearest-neighbor interactions and fixed boundary conditions. We first found that, for symmetric arrays, where all dimers have equal pumping rates and frequencies, fixing the internal coupling $\eta_{i n}$ constant just above Hopf bifurcation points leads to nearly equal amplitude oscillations (NEAO) of the electric fields, which persist even for external coupling $\eta_{e x}$

RUSSIAN JOURNAL OF NONLINEAR DYNAMICS, 2019, 15(4), 429-455 
nearly equal to $\eta_{i n}$. We then carried out similar experiments with arrays of 3, 5, 30 dimers (and beyond) and found interdimer coupling regimes, where NEAO are observed for significant ranges of parameters, even in the presence of small nonzero detuning and weak noise.

Studying asymmetric settings, characterized by different pumping rates, we discovered that dimer arrays still execute stable oscillations, but with one important difference: Some of the lasers are now seen to perform small oscillations about phase-locked states (SOPLS), while others exhibit NEAO. These collective oscillatory phenomena are robust for small nonzero detuning and weak noise effects, under fixed as well as periodic boundary conditions. Thus, our results suggest that laser dimer arrays need to be studied further and for widely different configurations, since they give rise to stable electric field oscillations that can be useful for optical communications, as well as many other applications in photonics.

Encouraged by our results so far, we have begun to study very recently periodic boundary conditions on a network of dimers, and have divided the dimers in two subpopulations having high and low pumping rates, respectively. We have thus observed for the first time a path to what we might call superradiance, where the maximum output power scales with the square of the number of elements in the array. This results from the subpopulation with the higher pumping rates, while the other population imposes large oscillations on the temporal mean value intensity of the whole system. Furthermore, this path to superradiance is very sensitive to the choice of initial conditions, but robust under small amount of detuning between the frequencies of each laser.

In fact, we have noticed that, by varying the coupling between the dimers, it is possible to change a stable out-of-phase state of our arrays into a stable in-phase state, using two subpopulations where each laser with high pumping rate is coupled to its neighbours with low pumping rates. This particular geometry has also been investigated theoretically and experimentally for a $\mathrm{Su}$-Schrieffer-Heeger (SSH) active microring resonator arrays by Parto et al. [46] where the judicious use of non-Hermiticity was shown to promote single edge-mode lasing in such arrays. We have also observed, after a transition to the in-phase stable state and for an asymmetric pumping distribution between the two subpopulations, that fully coherent emission is possible from the sublattice with the higher pumping rate.

We have also observed partial coherence intensity in different structures of laser arrays. Coherent radiation has already been achieved through the use of an external optical cavity for three homostructure GaAs lasers [60], combining single mode lasers using coupled cavities [56] and, more recently, in vertical cavity surface emitting laser (VCSEL) hexagonal arrays [58, 59]. In our system, however, full coherent emission from one of the subpopulations comes from the collective dynamics of the system due to the asymmetric distribution of the pumping rates throughout the array.

Currently, we are studying this form of superradiance in our dimer networks in more detail, concerning optical frequency detuning. Preliminary results indicate that, for small detuning, superradiance behavior is robust and the behavior is similar to the case of zero detuning. For larger detuning, however, there is no fixed phase relation between the oscillators and the system is incoherent.

\section{Acknowledgments}

We acknowledge the help of Dr. Kaloudis, who performed some of the computations and provided the corresponding figures in Section 5. 


\section{References}

[1] Johnson, M. T., Siriani, D. F., Peun Tan, M., and Choquette, K. D., Beam Steering via Resonance Detuning in Coherently Coupled Vertical Cavity Laser Arrays, Appl. Phys. Lett., 2013, vol. 103, no. 20, 201115, 4 pp.

[2] Fryslie, S.T.M., Johnson, M.T., and Choquette, K.D., Coherence Tuning in Optically Coupled Phased Vertical Cavity Laser Arrays, IEEE J. Quantum Electron., 2015, vol.51, no. 11, Art. No. 2600206, 6 pp.

[3] Wang, S. S. and Winful, H. G., Dynamics of Phase-Locked Semiconductor Laser Arrays, Appl. Phys. Lett., 1988, vol. 52, no. 21, pp. 1774-1776.

[4] Winful, H. G. and Rahman, L., Synchronized Chaos and Spatiotemporal Chaos in Arrays of Coupled Lasers, Phys. Rev. Lett., 1990, vol. 65, no. 13, pp. 1575-1578.

[5] Otsuka, K., Self-Induced Phase Turbulence and Chaotic Itinerancy in Coupled Laser Systems, Phys. Rev. Lett., 1990, vol. 65, no. 3, pp. 329-332.

[6] Winful, H. G., Instability Threshold for an Array of Coupled Semiconductor Lasers, Phys. Rev. A, 1992, vol. 46, no. 9, pp. 6093-6094.

[7] Rogister, F. and Roy, R., Localized Excitations in Arrays of Synchronized Laser Oscillators, Phys. Rev. Lett., 2007, vol. 98, no. 10, 104101, 4 pp.

[8] Soriano, M. C., García-Ojalvo, J., Mirasso, C.R., and Fischer, I., Complex Photonics: Dynamics and Applications of Delay-Coupled Semiconductors Lasers, Rev. Mod. Phys., 2013, vol. 85, no. 1, pp. $421-470$.

[9] Shena, J., Hizanidis, J., Kovanis, V., and Tsironis, G. P., Turbulent Chimeras in Large Semiconductor Laser Arrays, Sci. Rep., 2017, vol.7, Art. No.42116, 8 pp.

[10] Erneux, T. and Glorieux, P., Laser Dynamics, Cambridge: Cambridge Univ. Press, 2010.

[11] Valagiannopoulos, C. A. and Kovanis, V., Judicious Distribution of Laser Emitters to Shape the Desired Far-Field Patterns, Phys. Rev. A, 2017, vol. 95, no. 6, 063806, 7 pp.

[12] Yamamoto, Y., Takata, K., and Utsunomiya, S., Quantum Computing vs. Coherent Computing, New Generat. Comput., 2012, vol. 30, no. 4, pp. 327-355.

[13] Utsunomiya, S., Namekata, N., Takata, K., Akamatsu, D., Inoue, S., and Yamamoto, Y., Binary Phase Oscillation of Two Mutually Coupled Semiconductor Lasers, Opt. Express, 2015, vol. 23, no. 5, pp. 6029-6040.

[14] Gao, Z., Fryslie, S. T. M., Thompson, B. J., Carney, P. S., and Choquette, K. D., Parity-Time Symmetry in Coherently Coupled Vertical Cavity Laser Arrays, Optica, 2017, vol. 4, no. 3, pp. 323-329.

[15] Ge, L. and El-Ganainy, R., Nonlinear Modal Interactions in Parity-Time (PT) Symmetric Lasers, Sci. Rep., 2016, vol. 6, Art. No. 24889, 11 pp.

[16] Hodaei, H., Miri, M.-A., Heinrich, M., Christodoulides, D. N., and Khajavikhan, M., Parity-TimeSymmetric Microring Lasers, Science, 2014, vol. 346, no. 6212, pp. 975-978.

[17] Hodaei, H., Hassan, A. U., Ren, J., Hayenga, W. E., Miri, M.-A., Christodoulides, D. N., and Khajavikhan, M., Design Considerations for Single Mode Microring Lasers Using Parity-Time-Symmetry, IEEE J. Sel. Top. Quantum Electron., 2016, vol. 22, no. 5, Art. No. 1500307, 7 pp.

[18] Liertzer, M., Ge, L., Cerjan, A., Stone, A. D., Türeci, H. E., and Rotter, S., Pump-Induced Exceptional Points in Lasers, Phys. Rev. Lett., 2012, vol. 108, no. 17, 173901, 5 pp.

[19] El-Ganainy, R., Makris, K. G., Christodoulides, D. N., and Musslimani, Z. H., Theory of Coupled Optical PT-Symmetric Structures, Opt. Lett., 2007, vol. 32, no. 17, pp. 2632-2634.

[20] Guo, A., Salamo, G. J., Duchesne, D., Morandotti, R., Volatier-Ravat, M., Aimez, V., Siviloglou, G.A., and Christodoulides, D. N., Observation of $\mathcal{P} \mathcal{T}$-Symmetry Breaking in Complex Optical Potentials, Phys. Rev. Lett., 2009, vol. 103, no. 9, 093902, 4 pp.

[21] Makris, K. G., El-Ganainy, R., Christodoulides, D. N., and Musslimani, Z. H., PT-Symmetric Optical Lattices, Phys. Rev. A, 2010, vol.81, no. 6, 063807, 10 pp.

RUSSIAN JOURNAL OF NONLINEAR DYNAMICS, 2019, 15(4), 429-455 
[22] Rüter, Ch.E., Makris, K. G., El-Ganainy, R., Christodoulides, D. N., Segev, M., and Kip, D., Observation of Parity-Time Symmetry in Optics, Nat. Phys., 2010, vol. 6, pp. 192-195.

[23] Kottos, T., Optical Physics: Broken Symmetry Makes Light Work, Nat. Phys., 2010, vol. 6, $166-167$.

[24] Konotop, V.V., Yang, J., and Zezyulin, D. A., Nonlinear Waves in $\mathcal{P} \mathcal{T}$-Symmetric Systems, Rev. Mod. Phys., 2016, vol.88, no. 3, 035002, 59 pp.

[25] Zhou, X. and Chong, Y.D., PT-Symmetry Breaking and Nonlinear Optical Isolation in Coupled Microcavities, Opt. Express, 2016, vol.24, no. 7, pp. 6916-6930.

[26] Ramezani, H., Kottos, T., El-Ganainy, R., and Christodoulides, D. N., Unidirectional Nonlinear $\mathcal{P}$ T-Symmetric Optical Structures, Phys. Rev. A, 2010, vol. 82, no. 4, 043803, 6 pp.

[27] Kominis, Y., Bountis, T., and Flach, S., The Asymmetric Active Coupler: Stable Nonlinear Supermodes and Directed Transport, Sci. Rep., 2016, vol.6, Art. No. 33699, 7 pp.

[28] Kominis, Y., Bountis, T., and Flach, S., Stability through Asymmetry: Modulationally Stable Nonlinear Supermodes of Asymmetric Non-Hermitian Optical Couplers, Phys. Rev. A, 2017, vol. 95, no. 6, 063832, 6 pp.

[29] Winful, H. G. and Wang, S. S., Stability of Phase Locking in Coupled Semiconductor Laser Arrays, Appl. Phys. Lett., 1988, vol. 53, no. 20, pp. 1894-1896.

[30] Yanchuk, S., Schneider, K. R., and Recke, L., Dynamics of Two Mutually Coupled Semiconductor Lasers: Instantaneous Coupling Limit, Phys. Rev. E, 2004, vol.69, no. 5, 056221, 12 pp.

[31] Kuske, R. and Erneux, T., Localized Synchronization of Two Coupled Solid State Lasers, Opt. Commun., 1997, vol. 139, nos. 1-3, pp. 125-131.

[32] Hohl, A., Gavrielides, A., Erneux, T., and Kovanis, V., Localized Synchronization in Two Coupled Nonidentical Semiconductor Lasers, Phys. Rev. Lett., 1997, vol. 78, no. 25, pp. 4745-4748.

[33] Aronson, D. G., Ermentrout, G. B., and Kopell, N., Amplitude Response of Coupled Oscillators, Phys. D, 1990, vol. 41, no. 3, pp. 403-449.

[34] Hecht, E., Optics, 4th ed., San Francisco: Addison-Wesley, 2001.

[35] Gao, Z., Johnson, M. T., and Choquette, K. D., Rate Equation Analysis and Non-Hermiticity in Coupled Semiconductor Laser Arrays, J. Appl. Phys., 2018, vol. 123, no. 17, 173102, 11 pp.

[36] Kominis, Y., Kovanis, V., and Bountis, T., Spectral Signatures of Exceptional Points and Bifurcations in the Fundamental Active Photonic Dimer, Phys. Rev. A, 2017, vol. 96, no. 5, 053837, 5 pp.

[37] Kominis, Y., Kovanis, V., and Bountis, T., Controllable Asymmetric Phase Locked States of the Fundamental Active Photonic Dimer, Phys. Rev. A, 2017, vol. 96, no. 4, 043836, 9 pp.

[38] Papoulis, A. and Pillai, S. U., Probability, Random Variables and Stochastic Processes, 2nd ed., New York: McGraw-Hill, 1984.

[39] Kominis, Y., Choquette, K. D., Bountis, A., and Kovanis, V., Exceptional Points in Two Dissimilar Coupled Diode Lasers, Appl. Phys. Lett., 2018, vol. 113, no. 8, 081103, 4 pp.

[40] Kominis, Y., Choquette, K.D., Kovanis, V., and Bountis, A., Antiresonances and Ultrafast Resonances in Coupled Twin Photonic Oscillator, IEEE Photonics J., 2019, vol. 11, no. 1, 6 pp.

[41] Kominis, Y., Kovanis, V., and Bountis, A., Radically Tunable Ultrafast Photonic Oscillators via Differential Pumping, arXiv:1911.04179 (2019).

[42] Hodaei, H., Hassan, A. U., Wittek, S., Garcia-Gracia, H., El-Ganainy, R., Christodoulides, D. N., and Khajavikhan, M., Enhanced Sensitivity at Higher-Order Exceptional Points, Nature, 2017, vol. 548, pp. 187-191.

[43] Ren, J., Hodaei, H., Harari, G., Hassan, A. U., Chow, W., Soltani, M., Christodoulides, D., and Khajavikhan, M., Ultrasensitive Micro-Scale Parity-Time-Symmetric Ring Laser Gyroscope, Opt. Lett., 2017, vol. 42, no. 8, pp. 1556-1559.

[44] Liu, Zh.-P., Zhang, J., Özdemir, Ş. K., Peng, B., Jing, H., Lü, X.-Y., Li, Ch.-W., Yang, L., Nori, F., and Liu, Y.-X., Metrology with $\mathcal{P} \mathcal{T}$-Symmetric Cavities: Enhanced Sensitivity near the $\mathcal{P} \mathcal{T}$-Phase Transition, Phys. Rev. Lett., 2016, vol. 117, no. 11, 110802, 6 pp. 
[45] Chen, W., Özdemir, Ş. K., Zhao, G., Wiersig, J., and Yang, L., Exceptional Points Enhance Sensing in an Optical Microcavity, Nature, 2017, vol. 548, pp. 192-195.

[46] Parto, M., Wittek, S., Hodaei, H., Harari, G., Bandres, M. A., Ren, J., Rechtsman, M. C., Segev, M., Christodoulides, D. N., and Khajavikhan, M., Edge-Mode Lasing in 1D Topological Active Arrays, Phys. Rev. Lett., 2018, vol. 120, no. 11, 113901, 6 pp.

[47] Wilson, G. A., DeFreez, R. K., and Winful, H. G., Modulation of Twin-Emitter Semiconductor Lasers beyond the Frequency of Relaxation Oscillations, Opt. Commun., 1991, vol. 82, nos. 3-4, pp. $293-297$.

[48] Wilson, G. A., DeFreez, R. K., and Winful, H. G., Modulation of Phased-Array Semiconductor Lasers at K-Band Frequencies, IEEE J. Quantum Electron., 1991, vol. 27, no. 6, pp. 1696-1704.

[49] Sames, C., Chibani, H., Hamsen, C., Altin, P. A., Wilk, T., and Rempe, G., Antiresonance Phase Shift in Strongly Coupled Cavity QED, Phys. Rev. Lett., 2014, vol.112, no. 4, 043601, 5 pp.

[50] Koschny, T., Markoš, P., Smith, D. R., and Soukoulis, C. M., Resonant and Antiresonant Frequency Dependence of the Effective Parameters of Metamaterials, Phys. Rev. E, 2003, vol.68, no. 6, 065602, $4 \mathrm{pp}$.

[51] Dilena, M. and Morassi, A., The Use of Antiresonances for Crack Detection in Beams, J. Sound Vibration, 2004, vol. 276, nos. 1-2, pp. 195-214.

[52] Usechak, N. G., Grupen, M., Naderi, N., Li, Y., Lester, L.F., and Kovanis, V., Modulation Effects in Multi-Section Semiconductor Lasers, in Proc. SPIE 7933, Physics and Simulation of Optoelectronic Devices XIX (San Francisco, Calif., 2011), 79331I, 11 pp.

[53] Glasser, L. A., A Linearized Theory for the Diode Laser in an External Cavity, IEEE J. Quantum Electron., 1980, vol. 16, no. 5, pp. 525-531.

[54] Pochet, M., Usechak, N. G., Schmidt, J., and Lester, L. F., Modulation Response of a Long-Cavity, Gain-Levered Quantum-Dot Semiconductor Laser, Opt. Express, 2014, vol. 22, no. 2, pp. 1726-1734.

[55] Zehnlé, V., Theoretical Model for Coupled Solid-State Lasers, Phys. Rev. A, 2000, vol. 62, no. 3, 033814, 10 pp.

[56] Kouznetsov, D., Bisson, J., Shirakawa, A., and Ueda, K., Limits of Coherent Addition of Lasers: Simple Estimate, Opt. Rev., 2005, vol. 12, no. 6, pp. 445-447.

[57] García-Ojalvo, J., Casademont, J., Torrent, M. C., Mirasso, C. R., and Sancho, J. M., Coherence and Synchronization in Diode-Laser Arrays with Delayed Global Coupling, Internat. J. Bifur. Chaos Appl. Sci. Engrg., 1999, vol. 9, no. 11, pp. 2225-2229.

[58] Fryslie, S. T. M., Gao, Z., Dave, H., Thompson, B. J., Lakomy, K., Lin, Sh., Decker, P. J., McElfresh, D. K., Schutt-Ainé, J.E., and Choquette, K. D., Modulation of Coherently Coupled Phased Photonic Crystal Vertical Cavity Laser Arrays, IEEE J. Sel. Top. Quantum Electron., 2017, vol. 23, no. 6, Art. No. 1700409, 9 pp.

[59] Xun, M., Xu, Ch., Deng, J., Xie, Y., Jiang, G., Wang, J., Xu, K., and Chen, H., Wide Operation Range In-Phase Coherently Coupled Vertical Cavity Surface Emitting Laser Array Based on Proton Implantation, Opt. Lett., 2015, vol. 40, no. 10, pp. 2349-2352.

[60] Philipp, R. and Elisabeth, M., Spatially Coherent Radiation from an Array of GaAs Lasers, Appl. Phys. Lett., 1975, vol. 26, no. 8, pp. 475-477. 